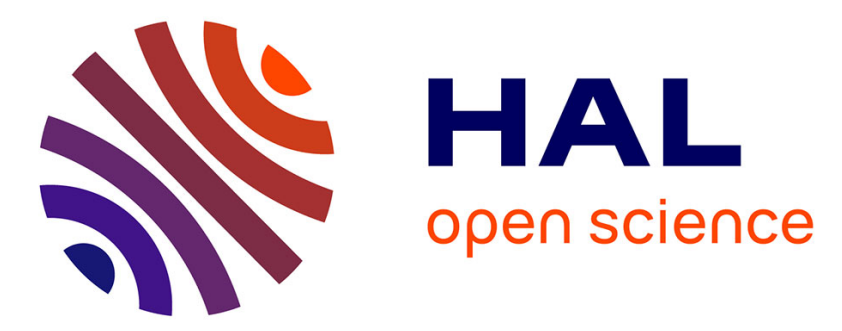

\title{
Locally implicit time integration strategies in a discontinuous Galerkin method for Maxwell's equations
}

Stéphane Descombes, Stéphane Lanteri, Ludovic Moya

\section{To cite this version:}

Stéphane Descombes, Stéphane Lanteri, Ludovic Moya. Locally implicit time integration strategies in a discontinuous Galerkin method for Maxwell's equations. [Research Report] RR-7983, INRIA. 2012, pp.28. hal-00702802v2

\section{HAL Id: hal-00702802 \\ https://hal.inria.fr/hal-00702802v2}

Submitted on 31 May 2012

HAL is a multi-disciplinary open access archive for the deposit and dissemination of scientific research documents, whether they are published or not. The documents may come from teaching and research institutions in France or abroad, or from public or private research centers.
L'archive ouverte pluridisciplinaire HAL, est destinée au dépôt et à la diffusion de documents scientifiques de niveau recherche, publiés ou non, émanant des établissements d'enseignement et de recherche français ou étrangers, des laboratoires publics ou privés. 


\section{Locally implicit time} integration strategies in a discontinuous Galerkin method for Maxwell's

\section{equations}

Stéphane Descombes, Stéphane Lanteri, Ludovic Moya

RESEARCH

REPORT

$\mathrm{N}^{\circ} 7983$

May 2012

Project-Team Nachos 



\title{
Giva
}

\section{Locally implicit time integration strategies in a discontinuous Galerkin method for Maxwell's equations}

\author{
Stéphane Descombes* Stéphane Lanteri ${ }^{\dagger}$, Ludovic Moya ${ }^{\dagger}$ \\ Project-Team Nachos \\ Research Report $\mathrm{n}^{\circ} 7983$ - May 2012 - 28 pages
}

\begin{abstract}
An attractive feature of discontinuous Galerkin (DG) spatial discretization is the possibility of using locally refined space grids to handle geometrical details. However, locally refined meshes lead to severe stability constraints on explicit integration methods to numerically solve a time-dependent partial differential equation. If the ratio of fine to coarse elements is small, the time step size restriction can be overcome by blending an implicit and an explicit scheme where only the solution variables living at fine elements are treated implicitly. The counterpart of this approach is having to solve a linear system per time step. But due to the assumed small fine to coarse elements ratio, the overhead will also be small while the solution can be advanced in time with step sizes determined by the coarse elements. In this paper, we present two locally implicit time integration methods for solving the time-domain Maxwell equations spatially discretized with a DG method. Numerical experiments for two-dimensional problems illustrate the theory and the usefulness of the implicit-explicit approaches in presence of local refinements.
\end{abstract}

Key-words: discontinuous Galerkin spatial discretization, locally implicit time integration methods, timedomain Maxwell equations

* University Nice Sophia Antipolis, J.A. Dieudonné Mathematics Laboratory, CNRS UMR 7351

${ }^{\dagger}$ Inria Sophia Antipolis - Méditerranée research center, Nachos project-team

\section{RESEARCH CENTRE}

SOPHIA ANTIPOLIS - MÉDITERRANÉE

2004 route des Lucioles - BP 93

06902 Sophia Antipolis Cedex 


\section{Méthode Galerkin discontinue et schémas d'intégration en temps localement implicites pour la résolution des équations de Maxwell}

Résumé : Les méthodes Galerkin discontinues sont particulièrement bien adaptées à la prise en compte de maillages localement raffinés, permettant de considérer avec précision des géométries complexes. Cependant, l'utilisation d'un schéma d'intégration en temps explicite conduit à une restriction importante du pas de temps admissible, ce dernier étant déterminé par les plus petits éléments du maillage considéré pour assurer la stabilité de la méthode. Si le rapport entre le nombre d'éléments fins et grossiers est petit, les restrictions de taille du pas de temps les plus contraignantes peuvent être évitées par l'utilisation d'un schéma d'intégration en temps localement implicite. La contre-partie est de devoir résoudre un système linéaire à chaque itération en temps. Cependant, le rapport entre le nombre d'éléments fins et grossiers étant petit alors le surcoût issu de la résolution du système linéaire sera faible, tandis que la solution peut être avancée avec un pas de temps déterminé par les éléments grossiers du maillage global. Dans ce papier, nous présentons deux méthodes d'intégration en temps localement implicites pour la résolution des équations de Maxwell en domaine temporel, discrétisées en espace par une méthode Galerkin discontinue. Des tests numériques sur des problèmes en dimension deux illustrent la théorie et l'utilité des approches implicite-explicite en présence de raffinements locaux.

Mots-clés : méthodes Galerkin discontinues, schéma d'intégration en temps localement implicite, équations de Maxwell en domaine temporel 


\section{Introduction}

The finite difference time-domain (FDTD) method based on Yee's scheme [24] is very often recognized as the most prominent method among physicists and engineers for the numerical resolution of time-domain Maxwell equations modeling electromagnetic wave propagation. The popularity of the FDTD method is mainly due to its simplicity in discretizing simple domain problems on one hand, and its easily achieved computational efficiency thanks to the use of fully explicit time stepping process. However its inability to effectively handle complex geometries has prompted the study of alternative methods. Also one of the main features of numerical methods based on finite element meshes like finite element (FETD), finite volume (FVTD) or discontinuous Galerkin time-domain (DGTD) methods is the possibility of using locally refined and non-conformal space grids to handle geometrical details. The first discontinuous Galerkin method was introduced in 1973 by Reed and Hill for solving the steady-state neutron transport equation [18]. Since then, this class of finite element methods has encountered an increasing interest and has been applied in many applications based on hyperbolic systems for acoustics, electromagnetics, elastodynamics, gaz dynamics and plasma dynamics applications.

DGTD methods are particularly well suited to the design of $h p$-adaptive strategies, i.e. strategies for which the characteristic mesh size $h$ and the interpolation degree $p$ change locally wherever it is needed. Then, in the presence of complex geometrical features, these methods appear as efficient strategies for the numerical solution of Maxwell's equations [8]. In this study the time-domain Maxwell equations are discretized in space using a nodal discontinuous Galerkin approach based on a discontinuous piecewise polynomial space for the numerical solution and the test functions. The method is detailed in Section 3 Theoretical analysis and numerous applications of nodal DG methods can be found for example in [4, 12] and more specifically for Maxwell's equations in [9, 11].

It is however well known that, when combined with an explicit time integration method to numerically solve a partial differential equation, a high order DG method can lead to a large step size restriction caused by the smallest grid elements. A possible alternative to overcome this limitation, generally induced by local mesh refinements in explicit methods, is to use smaller time steps, given by a local stability criterion, precisely where the smallest elements are located. The local character of DG methods is a very attractive feature for the development of explicit local time-stepping schemes. Such techniques have been developed for the second-order wave equations discretized in space by a DG method [6]. In [17], Piperno proposed a second-order symplectic local time-stepping scheme for Maxwell's equations in a non-conducting medium, based on the Störmer-Verlet method. Recently, Grote and Mitkova derived local time-stepping methods of arbitrarily high accuracy for Maxwell's equations from the standard leapfrog scheme [10]. In [19], Taube et al. also proposed an arbitrary high order local time-stepping method based on ADER DG approach for Maxwell's equation.

Another possibility to overcome step size restrictions is to use implicit-explicit (IMEX) schemes. IMEX methods were originally developed to solve problems with natural splittings into two parts. Such approaches are frequently used in Computational Fluid Dynamics [23]. The non-stiff part of the model equations is treated explicitly, for instance an advection term, and the stiff part implicitly, for instance a diffusion term or a term modeling stiff reactions. IMEX multistep methods were introduced by Crouzeix and Vara in [5, 20] for linear parabolic equations; high order combinations of explicit and diagonally implicit Runge-Kutta methods have been also proposed in [1, 3, 13, 14]. In particular, the authors of [13] defined the splitting such that the explicit set contains the coarsest elements, while the implicit set contains the smallest elements. Then, the most severe step size restrictions, imposed by the set treated explicitly, can be overcome. In the present study we consider two locally implicit time integration methods for Maxwell's equations. Starting from the well-suited leap-frog scheme for the explicit treatment, similarly to the splitting defined in [13], only solution variables associated to the smallest grid elements are treated implicitly. The counterpart of these approaches is the necessity to solve a linear system of equations at each time step. But if the ratio of fine to coarse elements is small, the overhead will also be small while 
the solution can be advanced in time with a step size only determined by the coarse elements. In contrast to the explicit local time-stepping methods, the critical time step is then unique and independent of the fine grid. The first locally implicit method, that we consider in Section 4.1 has been introduced by Piperno in [17]. This time integration scheme is a blend of the explicit second-order Leap-Frog scheme (LF2) and the implicit second-order Crank-Nicolson scheme (CN2), based on a nodal DG method for the spatial discretization. The second IMEX method which is treated in Section 4.2 has been proposed by Verwer in [21]. This method is also a combination of the LF2 and CN2 schemes, based on a clever component splitting which allows a significant gain in the sparsity of the matrix of the linear system enabling to solve this latter at lower cost. Altough the latter method is more general since the locally implicit scheme is directly formulated on the semi-discrete Maxwell equations, we propose to study this approach for a DG spatial discretization, since the local character of DG methods is particularly well suited to IMEX methods. We will notably state a sufficient condition for the stability derived from the conservation property presented in [21], and by adjusting the splitting, we will see that the two methods appear to be closely related. Finally we present in Section 5 numerical results for two-dimensional test problems. In particular, we are interested in the workload for the implicit treatment of each locally implicit method compared to the fully implicit approach (sparsity of matrices, memory requirements) and in the global efficiency of these methods compared to the fully explicit scheme (accuracy, CPU time gains).

\section{Problem statement}

We consider the time-domain Maxwell equations in three-dimensional space, that we write in the compact curl notation

$$
\left\{\begin{array}{l}
\varepsilon \partial_{t} \vec{E}=\operatorname{curl} \vec{H}-\sigma \vec{E}+\vec{J}^{E} \\
\mu \partial_{t} \vec{H}=-\operatorname{curl} \vec{E}
\end{array}\right.
$$

where $\vec{E}(\vec{x}, t)=\left(E^{x}, E^{y}, E^{z}\right)^{T}$ and $\vec{H}(\vec{x}, t)=\left(H^{x}, H^{y}, H^{z}\right)^{T}$ denote the electric and magnetic fields. $\varepsilon, \mu$ and $\sigma$ are (tensor) coefficients representing dielectric permittivity, magnetic permeability and conductivity, respectively. Finally $\vec{J}^{E}$ represents the imposed source current. These equations are set on a bounded three-dimensional spatial domain, denoted $\Omega$, with boundary $\partial \Omega=\Gamma_{m} \cup \Gamma_{a}$. We impose the following boundary conditions

$$
\left\{\begin{array}{l}
\vec{n} \times \vec{E}=0 \quad \text { on } \Gamma_{m}, \\
\vec{n} \times \vec{E}-\sqrt{\frac{\mu}{\varepsilon}} \vec{n} \times(\vec{H} \times \vec{n})=\vec{n} \times \vec{E}^{i n c}-\sqrt{\frac{\mu}{\varepsilon}} \vec{n} \times\left(\vec{H}^{i n c} \times \vec{n}\right) \quad \text { on } \Gamma_{a},
\end{array}\right.
$$

where $\vec{n}$ denotes the unit outward normal to $\partial \Omega$ and $\left(\vec{E}^{i n c}, \vec{H}^{i n c}\right)$ is a given incident field. The first boundary condition is called metallic (referring to a perfectly conduction surface) while the second one is called absorbing and takes the form of the Silver-Müller condition which is a first order approximation of the exact absorbing boundary condition.

\section{The DG discretization}

Let $\Omega_{h}$ be a partition of $\Omega$ into a set of $N_{h}$ tetrahedra $\tau_{i}$ of size $h_{i}$ with boundary $\partial \tau_{i}$

$$
\Omega \simeq \Omega_{h}=\bigcup_{i=1}^{N_{h}} \tau_{i}
$$


By convention $h$ denotes the maximum diameter of the (non-uniform) grid elements

$$
h=\max _{\tau_{i} \in \Omega_{h}} h_{i} .
$$

As in a finite element method for the given partition $\Omega_{h}$ we seek approximate electric and magnetic fields $\left(\vec{E}_{h}, \vec{H}_{h}\right)$ of $(\vec{E}, \vec{H})$ solution to the Maxwell equations (1) in a subspace $V_{h}$. We define the following finite dimensional subspace

$$
V_{h}=\left\{\vec{v}=\left(v_{1}, v_{2}, v_{3}\right)^{T} \in\left[L^{2}(\Omega)\right]^{3}: v_{k \mid \tau_{i}} \in \mathbb{P}_{p_{i}}\left(\tau_{i}\right), \quad \forall k, \quad \forall \tau_{i} \in \Omega_{h}\right\}
$$

where $\mathbb{P}_{p_{i}}\left(\tau_{i}\right)$ denotes the space of polynomial functions of degree at most $p_{i}$ inside the element $\tau_{i}$. Following the DG approach, inside each finite element $\tau_{i}$, the local electric and magnetic fields $\left(\left.\vec{E}_{h}\right|_{\tau_{i}},\left.\vec{H}_{h}\right|_{\tau_{i}}\right)=$ $\left(\vec{E}_{i}, \vec{H}_{i}\right)$ are expressed as linear combination of linearly independent basis vector $\Phi_{i l}, 1 \leq l \leq 3 d_{i}$

$$
\vec{E}_{i}(\vec{x}, t)=\sum_{l=1}^{3 d_{i}} E_{i l}(t) \Phi_{i l}(\vec{x}) \quad \text { and } \quad \vec{H}_{i}(\vec{x}, t)=\sum_{l=1}^{3 d_{i}} H_{i l}(t) \Phi_{i l}(\vec{x})
$$

where $d_{i}$ denotes the local number of degrees of freedom associated to the interpolation degree $p_{i}$ of $\tau_{i}$, i.e.

$$
d_{i}=\frac{\left(p_{i}+1\right)\left(p_{i}+2\right)\left(p_{i}+3\right)}{6}
$$

and $E_{i l}, H_{i l}$ reflect nodal values of $\vec{E}_{i}$ and $\vec{H}_{i}$, respectively. The global solution of Maxwell's equations (1) is then given by

$$
\vec{E}(\vec{x}, t) \simeq \vec{E}_{h}(\vec{x}, t)=\bigoplus_{i=1}^{N_{h}} \vec{E}_{i}(\vec{x}, t) \quad \text { and } \quad \vec{H}(\vec{x}, t) \simeq \vec{H}_{h}(\vec{x}, t)=\bigoplus_{i=1}^{N_{h}} \vec{H}_{i}(\vec{x}, t) .
$$

To avoid any ambiguity we specify the basis vector $\left(\Phi_{i l}\right)_{1<l<3 d_{i}}$ of the space $\left[\mathbb{P}_{p_{i}}\left(\tau_{i}\right)\right]^{3}$ and the sequences of nodal values $\left(E_{i l}\right)_{1 \leq l \leq 3 d_{i}}$ and $\left(H_{i l}\right)_{1 \leq l \leq 3 d_{i}}$. We denote by $\left(\varphi_{i l}\right)_{1 \leq l \leq d_{i}}$ the basis of the polynomial functions space $\mathbb{P}_{p_{i}}\left(\tau_{i}\right)$, then we define

$$
\left(\Phi_{i l}\right)_{1 \leq l \leq 3 d_{i}}=\left(\left(\begin{array}{c}
\varphi_{i 1} \\
0 \\
0
\end{array}\right),\left(\begin{array}{c}
0 \\
\varphi_{i 1} \\
0
\end{array}\right),\left(\begin{array}{c}
0 \\
0 \\
\varphi_{i 1}
\end{array}\right), \ldots,\left(\begin{array}{c}
\varphi_{i d_{i}} \\
0 \\
0
\end{array}\right),\left(\begin{array}{c}
0 \\
\varphi_{i d_{i}} \\
0
\end{array}\right),\left(\begin{array}{c}
0 \\
0 \\
\varphi_{i d_{i}}
\end{array}\right)\right) .
$$

Regarding $\left(E_{i l}\right)_{1 \leq l \leq 3 d_{i}}$ we order the sequence as follow

$$
\left(E_{i l}\right)_{1 \leq l \leq 3 d_{i}}=\left(E_{i 1}^{x}, E_{i 1}^{y}, E_{i 1}^{z}, \ldots, E_{i d_{i}}^{x}, E_{i d_{i}}^{y}, E_{i d_{i}}^{z}\right)
$$

and similarly for the sequence $\left(H_{i l}\right)_{1 \leq l \leq 3 d_{i}}$.

We now derive the DG spatial discretization. To simplify the presentation we assume that the given source current is equal to zero. First we introduce some notations. For each $\tau_{i}, \varepsilon_{i}, \mu_{i}$ and $\sigma_{i}$ denote respectively the local electric permittivity, magnetic permeability of the medium and the conduction coefficient, which are assumed constant inside the element $\tau_{i}$. For two distinct tetrahedra $\tau_{i}$ and $\tau_{k}$ in $\Omega_{h}$, the intersection $\tau_{i} \cap \tau_{k}$ is a triangle $a_{i k}$ which we call interface. The unitary normal vector of the interface $a_{i k}$ is denoted $\vec{n}_{i k}$, oriented from $\tau_{i}$ to $\tau_{k}$. For the boundary interface, the index $k$ corresponds to a fictitious element outside the domain. Finally, $v_{i}$ denotes the set of indices of the elements which have a common interface with $\tau_{i}$. Following the DG approach we now establish local variational formulations on each element 
of the space grid. Dot-multiplying (1) by any given vector $\Phi \in \operatorname{Span}\left(\Phi_{i j}, 1 \leq j \leq 3 d_{i}\right)$, integrating over each element $\tau_{i}$, integrating by part and finally replacing the exact fields $(\vec{E}, \vec{H})$ by the approximate fields $\left(\vec{E}_{h}, \vec{H}_{h}\right)$ yields

$$
\left\{\begin{array}{l}
\int_{\tau_{i}} \Phi \cdot \varepsilon_{i} \partial_{t} \vec{E}_{h} d \vec{x}-\int_{\tau_{i}} \operatorname{curl} \Phi \cdot \vec{H}_{h} d \vec{x}+\int_{\partial \tau_{i}} \Phi \cdot\left(\vec{H}_{h} \times \vec{n}\right) d s+\int_{\tau_{i}} \Phi \cdot \sigma_{i} \vec{E}_{h} d \vec{x}=0 \\
\int_{\tau_{i}} \Phi \cdot \mu_{i} \partial_{t} \vec{H}_{h} d \vec{x}+\int_{\tau_{i}} \operatorname{curl} \Phi \cdot \vec{E}_{h} d \vec{x}-\int_{\partial \tau_{i}} \Phi \cdot\left(\vec{E}_{h} \times \vec{n}\right) d s=0
\end{array}\right.
$$

One of the main features of the DG approach is that the approximate fields $\left(\vec{E}_{h}, \vec{H}_{h}\right)$ are allowed to be discontinuous across element boundaries. Then for such discontinuous fields we must define an approximate trace (i.e. a numerical trace) to evaluate the integrals over $\partial \tau_{i}$. In this study, we choose to use a centered approximation

$$
\forall i, \forall k \in v_{i},\left.\quad \vec{E}_{h}\right|_{a_{i k}}=\frac{\left.\vec{E}_{i}\right|_{a_{i k}}+\left.\vec{E}_{k}\right|_{a_{i k}}}{2} \quad \text { and }\left.\quad \vec{H}_{h}\right|_{a_{i k}}=\frac{\left.\vec{H}_{i}\right|_{a_{i k}}+\left.\vec{H}_{k}\right|_{a_{i k}}}{2} .
$$

Then for each face on the boundary of $\Omega_{h}, a_{i k} \in \Gamma_{m} \cup \Gamma_{a}$, the trace of a fictitious neighboring element is needed for the computation of the numerical flux. We treat the boundary conditions defined in (2) in a weak sense by defining appropriate values of the electric and magnetic fields in the fictitious element

$$
\begin{aligned}
& \forall a_{i k} \in \Gamma_{m}\left\{\begin{array}{l}
\left.\vec{E}_{k}\right|_{a_{i k}}=-\left.\vec{E}_{i}\right|_{a_{i k}}, \\
\left.\vec{H}_{k}\right|_{a_{i k}}=\left.\vec{H}_{i}\right|_{a_{i k}},
\end{array}\right. \\
& \forall a_{i k} \in \Gamma_{a}\left\{\begin{array}{l}
\left.\vec{E}_{k}\right|_{a_{i k}}=\sqrt{\frac{\mu_{i}}{\varepsilon_{i}}}\left(\left.\vec{H}_{i}\right|_{a_{i k}} \times \vec{n}_{i k}\right)+\left.\vec{E}_{i}^{i n c}\right|_{a_{i k}}-\sqrt{\frac{\mu_{i}}{\varepsilon_{i}}}\left(\left.\vec{H}_{i}^{i n c}\right|_{a_{i k}} \times \vec{n}_{i k}\right), \\
\left.\vec{H}_{k}\right|_{a_{i k}}=-\sqrt{\frac{\varepsilon_{i}}{\mu_{i}}}\left(\left.\vec{E}_{i}\right|_{a_{i k}} \times \vec{n}_{i k}\right)+\left.\vec{H}_{i}^{i n c}\right|_{a_{i k}}+\sqrt{\frac{\varepsilon_{i}}{\mu_{i}}}\left(\left.\vec{E}_{i}^{i n c}\right|_{a_{i k}} \times \vec{n}_{i k}\right) .
\end{array}\right.
\end{aligned}
$$

Now using $\left(\left.\vec{E}_{h}\right|_{\tau_{i}},\left.\vec{E}_{h}\right|_{\tau_{i}}\right)=\left(\vec{E}_{i}, \vec{H}_{i}\right)$ for volume integrals, evaluating the surface integrals in (11) with the centered numerical flux 12 and re-integrating by parts yields

$$
\left\{\begin{aligned}
\int_{\tau_{i}} \Phi \cdot \varepsilon_{i} \partial_{t} \vec{E}_{i} d \vec{x} & =\frac{1}{2} \int_{\tau_{i}}\left(\operatorname{curl} \Phi \cdot \vec{H}_{i}+\operatorname{curl} \vec{H}_{i} \cdot \Phi\right) d \vec{x}-\frac{1}{2} \sum_{k \in v_{i}} \int_{a_{i k}} \Phi \cdot\left(\vec{H}_{k} \times \vec{n}_{i k}\right) d s \\
& -\int_{\tau_{i}} \Phi \cdot \sigma_{i} \vec{E}_{i} d \vec{x} \\
\int_{\tau_{i}} \Phi \cdot \mu_{i} \partial_{t} \vec{H}_{i} d \vec{x} & =-\frac{1}{2} \int_{\tau_{i}}\left(\operatorname{curl} \Phi \cdot \vec{E}_{i}+\operatorname{curl} \vec{E}_{i} \cdot \Phi\right) d \vec{x}+\frac{1}{2} \sum_{k \in v_{i}} \int_{a_{i k}} \Phi \cdot\left(\vec{E}_{k} \times \vec{n}_{i k}\right) d s .
\end{aligned}\right.
$$

The system of equations (14) can be written in terms of scalar unknowns. According to the decomposition (6) of the local electric and magnetic fields $\left(\vec{E}_{i}, \vec{H}_{i}\right)$, denoting the column vectors $\left(E_{i l}\right)_{1 \leq l \leq 3 d_{i}}$ and $\left(H_{i l}\right)_{1 \leq l \leq 3 d_{i}}$ by $E_{i}$ and $H_{i}$, respectively, and replacing $\Phi$ by the basis vectors $\left(\Phi_{i j}\right)_{1 \leq j \leq 3 d_{i}}$, we obtain the equivalent system

$$
\left\{\begin{array}{l}
M_{i}^{\varepsilon} \partial_{t} E_{i}=K_{i} H_{i}-\sum_{k \in v_{i}} S_{i k} H_{k}-D_{i} E_{i} \\
M_{i}^{\mu} \partial_{t} H_{i}=-K_{i} E_{i}+\sum_{k \in v_{i}} S_{i k} E_{k}
\end{array}\right.
$$

where 
- $M_{i}^{\varsigma}$ ( $\varsigma$ stands for $\varepsilon$ or $\mu$ ) are the symmetric, positive definite mass matrices

$$
\left(M_{i}^{\varsigma}\right)_{j l}=\int_{\tau_{i}} \Phi_{i j}^{T} \varsigma_{i} \Phi_{i l} d \vec{x} \quad\left(1 \leq j, l \leq 3 d_{i}\right),
$$

- $K_{i}$ is the symmetric stiffness matrix

$$
\left(K_{i}\right)_{j l}=\frac{1}{2} \int_{\tau_{i}}\left(\Phi_{i j}^{T} \operatorname{curl} \Phi_{i l}+\Phi_{i l}^{T} \operatorname{curl} \Phi_{i j}\right) d \vec{x} \quad\left(1 \leq j, l \leq 3 d_{i}\right),
$$

- $S_{i k}$ are the rectangular interface matrices

$$
\left(S_{i k}\right)_{j l}=\frac{1}{2} \int_{a_{i k}} \Phi_{i j}^{T}\left(\Phi_{k l} \times \vec{n}_{i k}\right) d s \quad\left(1 \leq j \leq 3 d_{i}, 1 \leq l \leq 3 d_{k}\right) .
$$

- $D_{i}$ is the symmetric, positive semi-definite conduction matrix

$$
\left(D_{i}\right)_{j l}=\int_{\tau_{i}} \Phi_{i j}^{T} \sigma_{i} \Phi_{i l} d \vec{x} \quad\left(1 \leq j, l \leq 3 d_{i}\right)
$$

Taking into account the trace of fictitious neighboring elements (13) we can rewrite the local system of ODEs $[15$ as

$$
\left\{\begin{array}{l}
M_{i}^{\varepsilon} \partial_{t} E_{i}=K_{i} H_{i}-\sum_{a_{i k} \in \mathscr{F}_{i}^{i}} S_{i k} H_{k}-\sum_{a_{i k} \in \mathscr{F}_{i}^{m}} S_{i k}^{m} H_{i}+\sum_{a_{i k} \in \mathscr{F}_{i}^{a}}\left(S_{i k}^{E} E_{i}-X_{i k}^{E} W_{i}^{i n c}\right)-D_{i} E_{i}, \\
M_{i}^{\mu} \partial_{t} H_{i}=-K_{i} H_{i}+\sum_{a_{i k} \in \mathscr{F}_{i}^{i}} S_{i k} E_{k}-\sum_{a_{i k} \in \mathscr{F}_{i}^{m}} S_{i k}^{m} H_{i}+\sum_{a_{i k} \in \mathscr{F}_{i}^{a}}\left(S_{i k}^{H} H_{i}-X_{i k}^{H} W_{i}^{i n c}\right),
\end{array}\right.
$$

where

- $\left(S_{i k}^{m}\right)_{j l}=\frac{1}{2} \int_{a_{i k}} \Phi_{i j}^{T}\left(\Phi_{i l} \times \vec{n}_{i k}\right) d s \quad\left(1 \leq j, l \leq 3 d_{i}\right)$,

- $\left(S_{i k}^{E}\right)_{j l}=\frac{1}{2} \sqrt{\frac{\varepsilon_{i}}{\mu_{i}}} \int_{a_{i k}} \Phi_{i j}^{T}\left(\left(\Phi_{i l} \times \vec{n}_{i k}\right) \times \vec{n}_{i k}\right) d s \quad\left(1 \leq j, l \leq 3 d_{i}\right)$,

- $\left(S_{i k}^{H}\right)_{j l}=\frac{1}{2} \sqrt{\frac{\mu_{i}}{\varepsilon_{i}}} \int_{a_{i k}} \Phi_{i j}^{T}\left(\left(\Phi_{i l} \times \vec{n}_{i k}\right) \times \vec{n}_{i k}\right) d s \quad\left(1 \leq j, l \leq 3 d_{i}\right)$,

- $W_{i}^{i n c}$ is the column vector $\left(E_{i}^{i n c}, H_{i}^{i n c}\right)^{T}$ of length $6 d_{i}$, and for $1 \leq j \leq 3 d_{i}$

$$
\begin{aligned}
& \left(X_{i k}^{E}\right)_{j l}=\left\{\begin{array}{l}
-\frac{1}{2} \sqrt{\frac{\varepsilon_{i}}{\mu_{i}}} \int_{a_{i k}} \Phi_{i j}^{T}\left(\left(\Phi_{i l} \times \vec{n}_{i k}\right) \times \vec{n}_{i k}\right) d s \quad\left(1 \leq l \leq 3 d_{i}\right), \\
\frac{1}{2} \int_{a_{i k}} \Phi_{i j}^{T}\left(\Phi_{i\left(l-3 d_{i}\right)} \times \vec{n}_{i k}\right) d s \quad\left(3 d_{i}+1 \leq l \leq 6 d_{i}\right),
\end{array}\right. \\
& \left(X_{i k}^{H}\right)_{j l}=\left\{\begin{array}{l}
-\frac{1}{2} \int_{a_{i k}} \Phi_{i j}^{T}\left(\Phi_{i l} \times \vec{n}_{i k}\right) d s \quad\left(1 \leq l \leq 3 d_{i}\right), \\
\frac{1}{2} \sqrt{\frac{\mu_{i}}{\varepsilon_{i}}} \int_{a_{i k}} \Phi_{i j}^{T}\left(\left(\Phi_{i\left(l-3 d_{i}\right)} \times \vec{n}_{i k}\right) \times \vec{n}_{i k}\right) d s \quad\left(3 d_{i}+1 \leq l \leq 6 d_{i}\right),
\end{array}\right.
\end{aligned}
$$


Finally the set of local semi-discrete system (15) for each finite element $\tau_{i}$ can be transformed into a global system. Gathering all electric and magnetic unknowns in column vectors of size $d=\sum_{i=1}^{N_{h}} d_{i}$, denoted $E$ and $H$, respectively, we write

$$
\left\{\begin{array}{l}
M^{\varepsilon} \partial_{t} E=K H-A H-B H+C^{E} E-X^{E} W^{i n c} \\
M^{\mu} \partial_{t} H=-K E+A E-B E+C^{H} H-X^{H} W^{i n c}
\end{array}\right.
$$

where

- $M^{\varsigma}$ and $K$ are $3 d \times 3 d$ block diagonal mass and stiffness matrices with diagonal blocks equal to $M_{i}^{\varsigma}$ and $K_{i}$, respectively. Then the matrices $M^{\varsigma}$ are symmetric, positive definite and $K$ is symmetric.

- $A$ is a $3 d \times 3 d$ block sparse matrix, whose non-zero blocks equal to $S_{i k}$ when $a_{i k}$ is an internal interface. From (18) and the equality $\vec{n}_{k i}=-\vec{n}_{i k}$ we can check that $S_{k i}=S_{i k}^{T}$ and then $A$ is symmetric

$$
\left(S_{k i}\right)_{l j}=\frac{1}{2} \int_{a_{i k}}-\Phi_{k l}^{T}\left(\Phi_{i j} \times \vec{n}_{i k}\right) d s=\frac{1}{2} \int_{a_{i k}} \Phi_{i j}^{T}\left(\Phi_{k l} \times \vec{n}_{i k}\right) d s=\left(S_{i k}\right)_{j l} .
$$

- $B$ is a $3 d \times 3 d$ block diagonal matrix, whose non-zero blocks equal to $S_{i k}^{m}$ when $a_{i k}$ is a metallic boundary face. In that case $\left(S_{i k}^{m}\right)_{j l}=-\left(S_{i k}^{m}\right)_{l j}$, then $S_{i k}^{m}=-\left(S_{i k}^{m}\right)^{T}$ and $B$ is skew-symmetric.

- $C^{E}$ and $C^{H}$ are $3 d \times 3 d$ block diagonal matrices, whose non-zero blocks equal to $S_{i k}^{E}$ and $S_{i k}^{H}$, respectively, when $a_{i k}$ is an absorbing boundary face.

- $W^{i n c}$ denotes the column vectors $\left(E_{i l}, H_{i l}\right)_{1 \leq l \leq 3 d_{i}}$ for $1 \leq i \leq N_{h} . X^{E}$ and $X^{H}$ are $3 d \times 6 d$ block diagonal matrices, whose non-zero blocks equal to $X_{i k}^{E}$ and $X_{i k}^{H}$, respectively, when $a_{i k}$ is an absorbing boundary face.

- $D$ is a $3 d \times 3 d$ positive semi-definite, block diagonal matrix with diagonal blocks equal to $D_{i}$.

Reintroducing the source terms, and setting $S=K-A-B$ the system (21) reads

$$
\left\{\begin{array}{l}
M^{\varepsilon} \partial_{t} E=S H+C^{E} E-X^{E} W^{i n c}-D E+f^{E} \\
M^{\mu} \partial_{t} H=-S^{T} E+C^{H} H-X^{H} W^{i n c}+f^{H}
\end{array}\right.
$$

For convenience of presentation, we assume from now that $\Gamma_{a}=\emptyset$ and similarly to [2, 16, 21] we introduce the Cholesky factorization of the mass matrices

$$
M^{\varepsilon}=L_{M^{\varepsilon}} L_{M^{\varepsilon}}^{T} \text { and } M^{\mu}=L_{M^{\mu}} L_{M^{\mu}}^{T}
$$

where $L_{M^{\varepsilon}}$ and $L_{M^{\mu}}$ are triangular matrices. Then by introducing the change of variables $\tilde{E}=L_{M^{\varepsilon}}^{T} E$ and $\tilde{H}=L_{M^{\mu}}^{T} H$ in 22, we write

$$
\left\{\begin{array}{l}
\partial_{t} \tilde{E}=\tilde{S} \tilde{H}-\tilde{D} \tilde{E}+\tilde{f}^{E} \\
\partial_{t} \tilde{H}=-\tilde{S}^{T} \tilde{E}+\tilde{f}^{H}
\end{array}\right.
$$

where

$$
\begin{array}{ll}
\tilde{S}=L_{M^{\varepsilon}}^{-1} S\left(L_{M^{\mu}}^{-1}\right)^{T}, & \tilde{D}=L_{M^{\varepsilon}}^{-1} D\left(L_{M^{\varepsilon}}^{-1}\right)^{T}, \\
\tilde{f}^{E}=L_{M^{\varepsilon}}^{-1} f^{E}, & \tilde{f}^{H}=L_{M^{\mu}}^{-1} f^{H},
\end{array}
$$


For convenience of notation and presentation we use the same notation in (22) and (24) i.e.

$$
\left\{\begin{array}{l}
\partial_{t} E=S H-D E+f^{E}, \\
\partial_{t} H=-S^{T} E+f^{H} .
\end{array}\right.
$$

The results obtained for (22) can always be carried over to (26) and vice versa. From now we will proceed with (26). Note that the matrix $S$ within (26) satisfies [16]

$$
S \sim \frac{1}{h}, \quad \text { for } h \rightarrow 0,
$$

and the conduction matrix $D$ within 26 is diagonal with non-negative entries.

\section{Time integration methods}

A popular time integration method for the semi-discrete Maxwell system (22) is the second order LeapFrog scheme (LF2) that we write in the three-stage form, emanating from Verlet's method, see [17]

$$
\left\{\begin{array}{l}
\frac{H^{n+1 / 2}-H^{n}}{\Delta t / 2}=-S^{T} E^{n}+f^{H}\left(t_{n}\right), \\
\frac{E^{n+1}-E^{n}}{\Delta t}=S H^{n+1 / 2}-\frac{1}{2} D\left(E^{n+1}+E^{n}\right)+\frac{1}{2}\left(f^{E}\left(t_{n+1}\right)+f^{E}\left(t_{n}\right)\right), \\
\frac{H^{n+1}-H^{n+1 / 2}}{\Delta t / 2}=-S^{T} E^{n+1}+f^{H}\left(t_{n+1}\right),
\end{array}\right.
$$

where $\Delta t=t_{n+1}-t_{n}$ denotes the time step size and upper indices refer to time levels, as usual. This method has consistency two, is explicit in $\tilde{S}$, conditionally stable with a critical time step size proportional to $h^{-1}$, determined by the smallest grid element, [2]

$$
\Delta t<\frac{2}{\sqrt{\rho\left(S S^{T}\right)}},
$$

with strict inequality for zero conduction $(D=0)$ and where $\rho$ denotes the spectral radius. Hence DG applied with its attractive feature of local grid refinement may lead to unduly step size restrictions.

An alternative to $(28)$ is the second order, unconditionally stable Crank-Nicolson method (CN2) that we write in the three-stage form

$$
\left\{\begin{array}{l}
\frac{H^{n+1 / 2}-H^{n}}{\Delta t / 2}=-S^{T} E^{n}+f^{H}\left(t_{n}\right), \\
\frac{E^{n+1}-E^{n}}{\Delta t}=S H^{n+1}-\frac{1}{2} D\left(E^{n+1}+E^{n}\right)+\frac{1}{2}\left(f^{E}\left(t_{n+1}\right)+f^{E}\left(t_{n}\right)\right), \\
\frac{H^{n+1}-H^{n+1 / 2}}{\Delta t / 2}=-S^{T} E^{n+1}+f^{H}\left(t_{n+1}\right),
\end{array}\right.
$$

which only differs in the middle stage in the time level for $H$. For consistency and stability we refer to [22]. The expense for the implicit computation is too large to consider (30) as an attractive alternative to [28, especially in 3D (see e.g. [22]).

If the ratio of fine to coarse elements is small the unduly step size restriction of (28) and the overhead of 30 can be overcome by blending the two methods yielding locally implicit approaches where only variables associated to the smallest grid elements are implicitly treated. 


\subsection{The locally implicit method from [17]}

The implicit-explicit method proposed in [17] is based on the following component splitting. The set of DG grid elements is divided into two subsets, one made of the smallest elements for implicit treatment and its complementary set for explicit treatment. Accordingly to this subdivision, the unknowns $E$ and $H$ are reordered as

$$
E=\left(\begin{array}{c}
E_{e} \\
E_{i}
\end{array}\right) \quad \text { and } H=\left(\begin{array}{c}
H_{e} \\
H_{i}
\end{array}\right)
$$

where the indices $i$ and $e$ are associated to the elements of the subsets treated implicitly and explicitly, respectively. In line with this splitting the semi-discrete curl operator $S$, the conduction matrix $D$ and the source terms $f^{E}$ and $f^{H}$ are written as

$$
S=\left(\begin{array}{cc}
S_{e} & -A_{e i} \\
-A_{i e} & S_{i}
\end{array}\right), \quad D=\left(\begin{array}{cc}
D_{e} & 0 \\
0 & D_{i}
\end{array}\right), \quad f^{E}=\left(\begin{array}{c}
f_{e}^{E} \\
f_{i}^{E}
\end{array}\right) \quad \text { and } \quad f^{H}=\left(\begin{array}{c}
f_{e}^{H} \\
f_{i}^{H}
\end{array}\right) .
$$

Inserting this splitting into the semi-discrete DG Maxwell system 26) we obtain the system of ODEs

$$
\left\{\begin{array}{l}
\partial_{t} E_{e}=S_{e} H_{e}-A_{e i} H_{i}-D_{e} E_{e}+f_{e}^{E}(t), \\
\partial_{t} E_{i}=S_{i} H_{i}-A_{i e} H_{e}-D_{i} E_{i}+f_{i}^{E}(t), \\
\partial_{t} H_{e}=-S_{e}^{T} E_{e}+A_{i e}^{T} E_{i}+f_{e}^{H}(t), \\
\partial_{t} H_{i}=-S_{i}^{T} E_{i}+A_{e i}^{T} E_{e}+f_{i}^{H}(t) .
\end{array}\right.
$$

The locally implicit time integration scheme from [17] is a blend of LF2 28) and CN2 [30) applied to 33 . It reads

$$
\begin{aligned}
& \left\{\begin{array}{l}
\frac{H_{e}^{n+1 / 2}-H_{e}^{n}}{\Delta t / 2}=-S_{e}^{T} E_{e}^{n}+A_{i e}^{T} E_{i}^{n}+f_{e}^{H}\left(t_{n}\right) \\
\frac{E_{e}^{n+1 / 2}-E_{e}^{n}}{\Delta t / 2}=S_{e} H_{e}^{n+1 / 2}-A_{e i} H_{i}^{n}-D_{e} E_{e}^{n}+f_{e}^{E}\left(t_{n}\right),
\end{array}\right. \\
& \left\{\begin{aligned}
\frac{E_{i}^{n+1}-E_{i}^{n}}{\Delta t} & =S_{i}\left(\frac{H_{i}^{n+1}+H_{i}^{n}}{2}\right)-A_{i e} H_{e}^{n+1 / 2}-D_{i}\left(\frac{E_{i}^{n+1}+E_{i}^{n}}{2}\right) \\
& +\frac{f_{i}^{E}\left(t_{n+1}\right)+f_{i}^{E}\left(t_{n}\right)}{2}, \\
\frac{H_{i}^{n+1}-H_{i}^{n}}{\Delta t} & =-S_{i}^{T}\left(\frac{E_{i}^{n+1}+E_{i}^{n}}{2}\right)+A_{e i}^{T} E_{e}^{n+1 / 2}+\frac{f_{i}^{H}\left(t_{n+1}\right)+f_{i}^{H}\left(t_{n}\right)}{2},
\end{aligned}\right. \\
& \left\{\begin{array}{l}
\frac{E_{e}^{n+1}-E_{e}^{n+1 / 2}}{\Delta t / 2}=S_{e} H_{e}^{n+1 / 2}-A_{e i} H_{i}^{n+1}-D_{e} E_{e}^{n+1}+f_{e}^{E}\left(t_{n+1}\right), \\
\frac{H_{e}^{n+1}-H_{e}^{n+1 / 2}}{\Delta t / 2}=-S_{e}^{T} E_{e}^{n+1}+A_{i e}^{T} E_{i}^{n+1}+f_{e}^{H}\left(t_{n+1}\right) .
\end{array}\right.
\end{aligned}
$$

\subsubsection{Computational work}

Note that for $n \geq 1$ the derivative evaluations of the second explicit advance in the third block of (34) can be copied to the first explicit advance in the first block at the next time step. Furthermore from the second 
block of (34) we write

$$
\begin{aligned}
\left(I+\frac{\Delta t}{2} D_{i}\right) E_{i}^{n+1}= & \left(I-\frac{\Delta t}{2} D_{i}\right) E_{i}^{n}+\frac{\Delta t}{2} S_{i} H_{i}^{n+1}+\frac{\Delta t}{2} S_{i} H_{i}^{n}-\Delta t A_{i e} H_{e}^{n+1 / 2} \\
& +\frac{\Delta t}{2}\left(f_{i}^{E}\left(t_{n}+1\right)+f_{i}^{E}\left(t_{n}\right)\right)
\end{aligned}
$$

and

$$
H_{i}^{n+1}=H_{i}^{n}-\frac{\Delta t}{2} S_{i}^{T}\left(E_{i}^{n+1}+E_{i}^{n}\right)+\Delta t A_{e i}^{T} E_{e}^{n+1 / 2}+\frac{\Delta t}{2}\left(f_{i}^{H}\left(t_{n+1}\right)+f_{i}^{H}\left(t_{n}\right)\right) .
$$

Then by multiplying the equation (36) by $S_{i}$ and inserting the obtained result in (35) we get

$$
\mathscr{M}_{1} E_{i}^{n+1}=b_{i}^{n+1},
$$

where

$$
\begin{aligned}
\mathscr{M}_{1}= & I+\frac{\Delta t}{2} D_{i}+\frac{\Delta t^{2}}{4} S_{i} S_{i}^{T}, \\
b_{i}^{n+1}= & \left(I-\frac{\Delta t}{2} D_{i}-\frac{\Delta t^{2}}{4} S_{i} S_{i}^{T}\right) E_{i}^{n}+\Delta t S_{i} H_{i}^{n}+\frac{\Delta t^{2}}{2} S_{i} A_{e i}^{T} E_{e}^{n+1 / 2}-\Delta t A_{i e} H_{e}^{n+1 / 2} \\
& +\frac{\Delta t}{2}\left(f_{i}^{E}\left(t_{n}+1\right)+f_{i}^{E}\left(t_{n}\right)\right)+\frac{\Delta t^{2}}{4} S_{i}\left(f_{i}^{H}\left(t_{n+1}\right)+f_{i}^{H}\left(t_{n}\right)\right) .
\end{aligned}
$$

Thus $E_{i}^{n+1}$ can be obtained from the linear system (37) and successively $H_{i}^{n+1}$ from (36). Note that $\mathscr{M}_{1}$ is a square, symmetric, positive definite matrix, with dimension the length of $E_{i}$. Consequently if the ratio of fine to coarse grid elements is small, the workload induced by the implicit computation will be also small. Finally note that the evaluation of $E_{e}^{n+1}$ in the third block of (34) is implicit in the conduction matrix $D_{e}$, but since $D_{e}$ is diagonal this renders no additional overhead.

\subsubsection{Stability and conservation properties}

For the stability analysis of this method we refer to [7]. The proof is based on the conservation of a quadratic form of the numerical unknowns $E_{e}^{n}, E_{i}^{n}, H_{e}^{n}$ and $H_{i}^{n}$, previously established in [17]. The authors of [7] show that under a condition on the the time step size this quadratic form is positive definite and thus represents a discrete form of the electromagnetic energy. Consequently with the non-dissipative nature of the method they can conclude that this condition is sufficient for the stability of the locally implicit time integration scheme.

\subsubsection{Convergence}

Regarding convergence to the true underlying PDE solution of the implicit-explicit method we have proven in [16] the Theorem 1. Let $H_{e}^{h}(t), H_{i}^{h}(t), E_{e}^{h}(t)$ and $E_{i}^{h}(t)$ denote the exact solutions of the Maxwell problem under consideration, restricted to the space grid i.e. the exact solutions of the system of ODEs 33

$$
\left\{\begin{aligned}
\partial_{t} E_{e} & =S_{e} H_{e}-A_{e i} H_{i}-D_{e} E_{e}+f_{e}^{E}(t)+\sigma_{E_{e}}^{h}(t), \\
\partial_{t} E_{i} & =S_{i} H_{i}-A_{i e} H_{e}-D_{i} E_{i}+f_{i}^{E}(t)+\sigma_{E_{i}}^{h}(t), \\
\partial_{t} H_{e} & =-S_{e}^{T} E_{e}+A_{i e}^{T} E_{i}+f_{e}^{H}(t)+\sigma_{H_{e}}^{h}(t), \\
\partial_{t} H_{i} & =-S_{i}^{T} E_{i}+A_{e i}^{T} E_{e}+f_{i}^{H}(t)+\sigma_{H_{i}}^{h}(t) .
\end{aligned}\right.
$$

with $\sigma_{E_{e}}^{h}(t), \sigma_{E_{i}}^{h}(t), \sigma_{H_{e}}^{h}(t)$ and $\sigma_{H_{i}}^{h}(t)$ standing for the spatial truncation errors. 
Theorem 1. Let $f^{H}(t), f^{E}(t) \in C^{2}[0, T]$ and suppose a Lax-Richtmyer stable space-time grid refinement $\Delta t \sim h, h \rightarrow 0$. On $[0, T]$, the approximations $H_{e}^{n}, H_{i}^{n}, E_{e}^{n}$ and $E_{i}^{n}$ resulting from method (34) then converge to $H_{e}^{h}(t), H_{i}^{h}(t), E_{e}^{h}(t)$ and $E_{i}^{h}(t)$

(i) at least at first order,

(ii) at least at second order, if in addition $A_{e i}^{T} S_{e} H_{e}^{h(1)}(t)=\mathscr{O}\left(\Delta t^{-1}\right)$ for $h \rightarrow 0$.

Then only the first-order convergence of method (34) is guaranteed and the component splitting can reduce the temporal convergence order for a simultaneous, stable space-time grid refinement. An attractive feature of the DG approach is the ability to easily increase the spatial convergence order. The reduction by one of the PDE order for the locally implicit method (34) raises the legitimate question of the efficiency of the method when high order approximation polynomials are used within the DG method. In [16] we have observed that for a given error the locally implicit method (34) needs a finer grid or significantly more DOF compared to the fully explicit method (28). In other words the method (34) needs more points per wavelength. Consequently a high order DG spatial discretization in the locally time integration scheme (34) becomes less appealing due to the errors introduced by the lower temporal order.

\subsection{The locally implicit method from [21]}

This implicit-explicit time integration method is also a blend of (28) and (30) applied to the generic semi-discrete Maxwell system 26]

$$
\left\{\begin{aligned}
\frac{H^{n+1 / 2}-H^{n}}{\Delta t / 2} & =-S^{T} E^{n}+f^{H}\left(t_{n}\right), \\
\frac{E^{n+1}-E^{n}}{\Delta t} & =S_{0} H^{n+1 / 2}+\frac{1}{2} S_{1}\left(H^{n}+H^{n+1}\right) \\
& -\frac{1}{2} D\left(E^{n}+E^{n+1}\right)+\frac{1}{2}\left(f^{E}\left(t_{n}\right)+f^{E}\left(t_{n+1}\right)\right), \\
\frac{H^{n+1}-H^{n+1 / 2}}{\Delta t / 2} & =-S^{T} E^{n+1}+f^{H}\left(t_{n+1}\right),
\end{aligned}\right.
$$

where $S=S_{0}+S_{1}$ is a matrix splitting. The method is implicit in $S_{1}$ and explicit in $S_{0}$. For $S_{0}=0$ we recover (30) and for $S_{1}=0$ the method 28).

\subsubsection{Computational work}

First note that for $n \geq 1$ the third stage derivative computation can be copied to the first stage at the next time step. Furthermore from the second and the third stage of (40) we write

$$
\begin{aligned}
\left(I+\frac{\Delta t}{2} D\right) E^{n+1}= & \left(I-\frac{\Delta t}{2} D\right) E^{n}+\frac{\Delta t}{2} S_{1} H^{n+1}+\frac{\Delta t}{2} S_{1} H^{n}-\Delta t S_{0} H^{n+1 / 2} \\
& +\frac{\Delta t}{2}\left(f^{E}\left(t_{n+1}\right)+f^{E}\left(t_{n}\right)\right),
\end{aligned}
$$

and

$$
H^{n+1}=H^{n+1 / 2}-\frac{\Delta t}{2} S^{T} E^{n+1}+\frac{\Delta t}{2} f^{H}\left(t_{n+1}\right) .
$$

Then by multiplying the equation (42) by $S_{1}$ and inserting the obtained result in (41) we get

$$
\mathscr{M}_{2} E^{n+1}=b_{n+1}
$$


where

$$
\begin{aligned}
\mathscr{M}_{2}= & I+\frac{\Delta t}{2} D+\frac{\Delta t^{2}}{4} S_{1} S^{T}, \\
b_{n+1}= & E^{n}+\Delta t S_{0} H^{n+1 / 2}+\frac{\Delta t}{2} S_{1}\left(H^{n}+H^{n+1 / 2}+\frac{\Delta t}{2} f^{H}\left(t_{n+1}\right)\right) \\
& -\frac{\Delta t}{2} D E^{n}+\frac{\Delta t}{2}\left(f^{E}\left(t_{n}\right)+f^{E}\left(t_{n+1}\right)\right) .
\end{aligned}
$$

Thus $E^{n+1}$ can be obtained from the linear system (43) and successively $H^{n+1}$ from (42). The initial splitting adopted in [21] is defined as follow

$$
S_{1}=S S_{H}
$$

where $S_{H}$ is a diagonal matrix of dimension the length of $H$ with

$$
\left(S_{H}\right)_{j j}=\left\{\begin{array}{l}
0, \text { component } H_{j} \text { of } H \text { to be treated explicitly, } \\
1, \text { component } H_{j} \text { of } H \text { to be treated implicitly. }
\end{array}\right.
$$

With this definition the author of [21] notes that $S_{1} S^{T}$ is symmetric, $S_{1} S^{T}=S S_{H} S^{T}=S S_{H} S_{H} S^{T}=S_{1} S_{1}^{T}$, which facilitates the resolution of 43 . The matrix $\mathscr{M}_{2}$ is then given by

$$
\mathscr{M}_{2}=I+\frac{\Delta t}{2} D+\frac{\Delta t^{2}}{4} S_{1} S_{1}^{T}
$$

For $S_{1}=S$ we recover the matrix of the linear system of the fully implicit method [30, [22]

$$
\mathscr{M}=I+\frac{\Delta t}{2} D+\frac{\Delta t^{2}}{4} S S^{T} .
$$

With the adopted splitting the matrix $\mathscr{M}_{2}$ will be significantly more sparse than without splitting. This gain of sparsity for the matrix $\mathscr{M}_{2}$ enables to solve the linear system at lower costs.

Note that the locally implicit methods (34) and (40) are very similar. Indeed, giving an equivalent formulation of (40) using $E^{n+1 / 2}$

$$
\left\{\begin{array}{l}
\frac{H^{n+1 / 2}-H^{n}}{\Delta t / 2}=-S^{T} E^{n}+f^{H}\left(t_{n}\right), \\
\frac{E^{n+1 / 2}-E^{n}}{\Delta t}=\frac{1}{2} S_{0} H^{n+1 / 2}+\frac{1}{2} S_{1} H^{n}-\frac{1}{2} D E^{n}+\frac{1}{2} f^{E}\left(t_{n}\right), \\
\frac{E^{n+1}-E^{n+1 / 2}}{\Delta t}=\frac{1}{2} S_{0} H^{n+1 / 2}+\frac{1}{2} S_{1} H^{n+1}-\frac{1}{2} D E^{n+1}+\frac{1}{2} f^{E}\left(t_{n+1}\right), \\
\frac{H^{n+1}-H^{n+1 / 2}}{\Delta t / 2}=-S^{T} E^{n+1}+f^{H}\left(t_{n+1}\right),
\end{array}\right.
$$

Adopting the subdivisions (31) - 32) we adjust the splitting (45) of the DG matrix S

$$
S_{1}=S S_{h}=\left(\begin{array}{cc}
S_{e} & -A_{e i} \\
-A_{i e} & S_{i}
\end{array}\right)\left(\begin{array}{cc}
0 & 0 \\
0 & I
\end{array}\right)=\left(\begin{array}{cc}
0 & -A_{e i} \\
0 & S_{i}
\end{array}\right),
$$

Hence,

$$
S_{0}=S-S_{1}=\left(\begin{array}{rr}
S_{e} & 0 \\
-A_{i e} & 0
\end{array}\right)
$$

$\operatorname{RR} n^{\circ} 7983$ 
Adopting the subdivisions 31 and considering the previous splitting into 49 we obtain the following locally implicit time integration method

$$
\begin{aligned}
& \left\{\begin{array}{l}
\frac{H_{e}^{n+1 / 2}-H_{e}^{n}}{\Delta t / 2}=-S_{e}^{T} E_{e}^{n}+A_{i e}^{T} E_{i}^{n}+f_{e}^{H}\left(t_{n}\right) \\
\frac{E_{e}^{n+1 / 2}-E_{e}^{n}}{\Delta t / 2}=S_{e} H_{e}^{n+1 / 2}-A_{e i} H_{i}^{n}-D_{e} E_{e}^{n}+f_{e}^{E}\left(t_{n}\right),
\end{array}\right. \\
& \left\{\begin{aligned}
\frac{E_{i}^{n+1}-E_{i}^{n}}{\Delta t} & =S_{i}\left(\frac{H_{i}^{n+1}+H_{i}^{n}}{2}\right)-A_{i e} H_{e}^{n+1 / 2}-D_{i}\left(\frac{E_{i}^{n+1}+E_{i}^{n}}{2}\right) \\
& +\frac{f_{i}^{E}\left(t_{n+1}\right)+f_{i}^{E}\left(t_{n}\right)}{2}, \\
\frac{H_{i}^{n+1}-H_{i}^{n}}{\Delta t} & =-S_{i}^{T}\left(\frac{E_{i}^{n+1}+E_{i}^{n}}{2}\right)+A_{e i}^{T}\left(\frac{E_{e}^{n+1}+E_{e}^{n}}{2}\right)+\frac{f_{i}^{H}\left(t_{n+1}\right)+f_{i}^{H}\left(t_{n}\right)}{2},
\end{aligned}\right. \\
& \left\{\begin{array}{l}
\frac{E_{e}^{n+1}-E_{e}^{n+1 / 2}}{\Delta t / 2}=S_{e} H_{e}^{n+1 / 2}-A_{e i} H_{i}^{n+1}-D_{e} E_{e}^{n+1}+f_{e}^{E}\left(t_{n+1}\right), \\
\frac{H_{e}^{n+1}-H_{e}^{n+1 / 2}}{\Delta t / 2}=-S_{e}^{T} E_{e}^{n+1}+A_{i e}^{T} E_{i}^{n+1}+f_{e}^{H}\left(t_{n+1}\right) .
\end{array}\right.
\end{aligned}
$$

Comparing this method with the locally implicit method (34), the only difference appears in the second block for the definition of the variable $H_{i}$, we read

$$
A_{e i}^{T}\left(\frac{E_{e}^{n+1}+E_{e}^{n}}{2}\right) \text { instead of } A_{e i}^{T} E_{e}^{n+1 / 2}
$$

Albeit this difference concerning the treatment of the interface matrix $A_{e i}^{T}$ seems minor, we will see that the consequences on the error behavior are significant, notably regarding convergence to the true underlying PDE solution; while the method (34) suffers from order reduction [16] this is not the case with the method (40) [21]. However we remark that the matrix $\mathscr{M}_{2}$ has dimension the length of $E$, while the matrix $\mathscr{M}_{1}$ has dimension the length of $E_{i}$. Hence the workload for solving the linear system of the method (34) is smaller. More precisely the linear system (43) of the method (40) is equivalent to

$$
\left(\begin{array}{cc}
I+\frac{\Delta t}{2} D_{e}+\frac{\Delta t^{2}}{4} A_{e i}^{T} A_{e i} & -\frac{\Delta t^{2}}{4} A_{e i} S_{i}^{T} \\
-\frac{\Delta t^{2}}{4} S_{i} A_{e i}^{T} & I+\frac{\Delta t}{2} D_{i}+\frac{\Delta t^{2}}{4} S_{i}^{T} S_{i}
\end{array}\right)\left(\begin{array}{c}
E_{e}^{n+1} \\
E_{i}^{n+1}
\end{array}\right)=\left(\begin{array}{c}
b_{e}^{n+1} \\
b_{i}^{n+1}
\end{array}\right)
$$

where $b_{e}^{n+1}, b_{i}^{n+1}$ collect available vectors. By omitting the identity matrix in the above $(2,2)$-block matrix and in the matrix $\mathscr{M}_{1}$ we expect that the overhead for solving the linear system of the method (40) will not be excessive compared to the method (34), because $A_{e i}^{T} A_{e i}, A_{e i} S_{i}^{T}$ and $S_{i} A_{e i}^{T}$ are very sparse block diagonal matrices.

\subsubsection{Stability and conservation properties}

In [21], the author shows that the locally implicit method (40) for time integration of the semi-discrete Maxwell system (26) exactly conserve the following quadratic form of the numerical unknowns $E^{n}$ and $H^{n}$

$$
\mathscr{E}_{n}=\left\|H^{n}\right\|_{2}^{2}+\left\|E^{n}\right\|_{2}^{2}-\frac{\Delta t^{2}}{4}\left\langle S_{0} S^{T} E^{n}, E^{n}\right\rangle,
$$


for zero damping matrix $D$. By definition of $S_{0}=S\left(I-S_{H}\right)$ the matrix $S_{0} S^{T}$ is symmetric, since $S_{0} S^{T}=$ $S\left(I-S_{h}\right) S^{T}=S\left(I-S_{H}\right)\left(I-S_{H}\right) S^{T}=S_{0} S_{0}^{T}$. Then we have

$$
\mathscr{E}_{n}=\left\|H^{n}\right\|_{2}^{2}+\left\|E^{n}\right\|_{2}^{2}-\frac{\Delta t^{2}}{4}\left\langle S_{0} S_{0}^{T} E^{n}, E^{n}\right\rangle
$$

The minus sign in front of the third term does not allow to conclude directly on the stability of the locally implicit time integration method [40). In [21] the author notes that for zero diagonal matrix $S_{H}$ one recover the conservation property of the explicit scheme (28) (see [2]) and since the entries of $S_{H}$ are either zero or one, the deviation from the exact energy is reduced for the method 40 compared to the method (28).

The condition under which $\mathscr{E}_{n}$ is a positive definite quadratic form of the numerical unknowns and thus represents a discrete form of the electromagnetic energy is not given in [21]. In the following, we state a condition on the time step $\Delta t$ such that $\mathscr{E}_{n}$ is a positive definite quadratic form.

Lemma 2. The quadratic form $\mathscr{E}_{n}$ given by 56 is a positive definite quadratic form of the numerical unknowns $E^{n}$ and $H^{n}$ if

$$
\Delta t<\frac{2}{\sqrt{\rho\left(S_{0} S_{0}^{T}\right)}},
$$

where $\rho$ denotes the spectral radius.

Proof. From (56) we have

$$
\begin{aligned}
\mathscr{E}_{n} & =\left\|H^{n}\right\|_{2}^{2}+\left\|E^{n}\right\|_{2}^{2}-\frac{\Delta t^{2}}{4}\left\langle S_{0} S_{0}^{T} E^{n}, E^{n}\right\rangle \\
& =\left\|H^{n}\right\|_{2}^{2}+\left\|E^{n}\right\|_{2}^{2}-\frac{\Delta t^{2}}{4}\left\|S_{0}^{T} E^{n}\right\|_{2}^{2} .
\end{aligned}
$$

Note that

$$
\left\|S_{0}^{T} E^{n}\right\|_{2} \leq\left\|S_{0}^{T}\right\|_{2}\left\|E^{n}\right\|_{2}=\sqrt{\rho\left(S_{0} S_{0}^{T}\right)}\left\|E^{n}\right\|_{2}
$$

hence

$$
\mathscr{E}_{n} \geq\left\|H^{n}\right\|_{2}^{2}+\left(1-\frac{\Delta t^{2}}{4} \rho\left(S_{0} S_{0}^{T}\right)\right)\left\|E^{n}\right\|_{2}^{2}
$$

allows to obtain that under the condition $(57), \mathscr{E}_{n}$ is a positive definite quadratic form of the numerical unknowns $E^{n}$ and $H^{n}$.

Consequently $\mathscr{E}_{n}$ represents a discrete form of the electromagnetic energy and with the conservation property (56) we can conclude that (57) states a sufficient condition for the stability of the locally implicit time integration method (40). Note that the condition (57) is similar to the stability condition of the LF2 scheme (29) with $S_{0}$ instead of $S$, allowing to let the definition $\Delta t$ be restricted to the subset of the coarse grid elements.

\subsubsection{Convergence}

In [21] the author has proven that the subdivision into coarse and fine elements is not detrimental to the second-order ODE convergence of the method (40), under stable simultaneous space-time grid refinement towards the true underlying PDE solution. Let $H^{h}(t)$ and $E^{h}(t)$ denote the exact solutions of the Maxwell problem under consideration, restricted to the space grid i.e. the exact solutions of the system of ODEs

$$
\left\{\begin{array}{l}
\partial_{t} E^{h}(t)=S H^{h}(t)-D E^{h}(t)+f^{E}(t)+\sigma_{E}^{h}(t), \\
\partial_{t} H^{h}(t)=-S^{T} E^{h}(t)+f^{H}(t)+\sigma_{H}^{h}(t),
\end{array}\right.
$$


with $\sigma_{H}^{h}(t)$ and $\sigma_{E}^{h}(t)$ denote the spatial truncation errors.

Theorem 3. Let $f^{H}(t), f^{E}(t) \in C^{2}[0, T]$ and suppose a Lax-Richtmyer stable space-time grid refinement $\Delta t \sim h, h \rightarrow 0$. On the interval $[0, T]$ the approximations $H^{n}$ and $E^{n}$ of method (40) then converge with temporal order two to $H^{h}(t)$ and $E^{h}(t)$.

\section{Numerical results for two-dimensional test problems}

\subsection{D TM Maxwell's equations}

Let $\varepsilon, \mu$ and $\sigma$ in (1) be scalar. Writing $E=\left(E^{x}, E^{y}, E^{z}\right)$ and $H=\left(H^{x}, H^{y}, H^{z}\right)$, in three dimension we then have

$$
\left\{\begin{aligned}
\mu \frac{\partial H^{x}}{\partial t} & =\frac{\partial E^{y}}{\partial z}-\frac{\partial E^{z}}{\partial y}, & \varepsilon \frac{\partial E^{x}}{\partial t} & =\frac{\partial H^{z}}{\partial y}-\frac{\partial H^{y}}{\partial z}-\sigma E^{x}-J_{E}^{x}, \\
\mu \frac{\partial H^{y}}{\partial t} & =\frac{\partial E^{z}}{\partial x}-\frac{\partial E^{x}}{\partial z}, & \varepsilon \frac{\partial E^{y}}{\partial t} & =\frac{\partial H^{x}}{\partial z}-\frac{\partial H^{z}}{\partial x}-\sigma E^{y}-J_{E}^{y}, \\
\mu \frac{\partial H^{z}}{\partial t} & =\frac{\partial E^{x}}{\partial y}-\frac{\partial E^{y}}{\partial z}, & \varepsilon \frac{\partial E^{z}}{\partial t} & =\frac{\partial H^{y}}{\partial x}-\frac{\partial H^{x}}{\partial y}-\sigma E^{z}-J_{E}^{z} .
\end{aligned}\right.
$$

From this three-dimensional model we derive the two-dimensional Transverse Magnetic model (TM) for components $H^{x}, H^{y}$ and $E^{z}$

$$
\left\{\begin{aligned}
\mu \frac{\partial H^{x}}{\partial t} & =-\frac{\partial E^{z}}{\partial y} \\
\mu \frac{\partial H^{y}}{\partial t} & =\frac{\partial E^{z}}{\partial x} \\
\varepsilon \frac{\partial E^{z}}{\partial t} & =\frac{\partial H^{y}}{\partial x}-\frac{\partial H^{x}}{\partial y}-\sigma E^{z}-J_{E}^{z}
\end{aligned}\right.
$$

In the following, the equations 63 are space discretized using a DG method formulated on triangular meshes. In the preliminary implementation of this DG method, the approximation of the electromagnetic field components within a triangle $\tau_{i}$ relies on a nodal $\mathbb{P}_{l}$ interpolation method. The a priori convergence analysis for this DGTD method based on a centered numerical flux and formulated on simplicial meshes shows that the convergence rate is $\mathscr{O}\left(h^{l}\right)$ for a $l$-th interpolation order [9]. A triangle $\tau_{i}$ is characterized by its height $h_{i}$. The critical step size, denoted $\Delta t_{c}$, used in the numerical tests is given by

$$
\Delta t_{c}=C F L \times h_{k}^{\min }
$$

where the parameter $h_{k}^{\min }$ characterizes the smallest height of a triangle $\tau_{k}$ inside the region treated explicitly. The values of the $C F L$ number, given in Table 1 . corresponds to the numerical stability, i.e. the limit beyond which we observe a growth of the discrete energy.

\begin{tabular}{lllll}
\hline Method & DGTD- $\mathbb{P}_{1}$ & DGTD- $\mathbb{P}_{2}$ & DGTD- $\mathbb{P}_{3}$ & DGTD- $\mathbb{P}_{4}$ \\
\hline Numerical $C F L$ & 0.30 & 0.20 & 0.13 & 0.09 \\
\hline
\end{tabular}

Table 1: Numerical value of the $C F L$ number in 64. 


\subsection{Model test problem with an exact solution}

We set $\varepsilon=\mu=1$ and $\sigma=0$ and we solve $(63)$ in an unitary cavity $\left(\Omega=[0,1]^{2}\right)$ with an artificial source term $J_{z}^{E}$ defined by

$$
J_{z}^{E}(x, y, t)=-e^{t}[x(1-x) y(1-y)+2 x(1-x)+2 y(1-y)] .
$$

The exact solution of this problem is given by

$$
\left\{\begin{array}{l}
E^{z}(x, y, t)=e^{t} x(1-x) y(1-y), \\
H^{x}(x, y, t)=-e^{t} x(1-x)(1-2 y), \\
H^{y}(x, y, t)=e^{t} y(1-y)(1-2 x) .
\end{array}\right.
$$

We impose a metallic boundary condition on the boundary of the cube (see (2)) such that the tangential component of the electric field vanishes on the boundaries

$$
n \times E^{z}=0 \text { on } \partial \Omega \text {, }
$$

where $n$ denoted the unit outw ard normal to $\partial \Omega$. Throughout this subsection the total simulation time $T$ is set to $T=5$. The source term $J_{z}^{E}$ and the electric field $E^{z}$ are represented on Figure 1 at the final simulation time $T$.
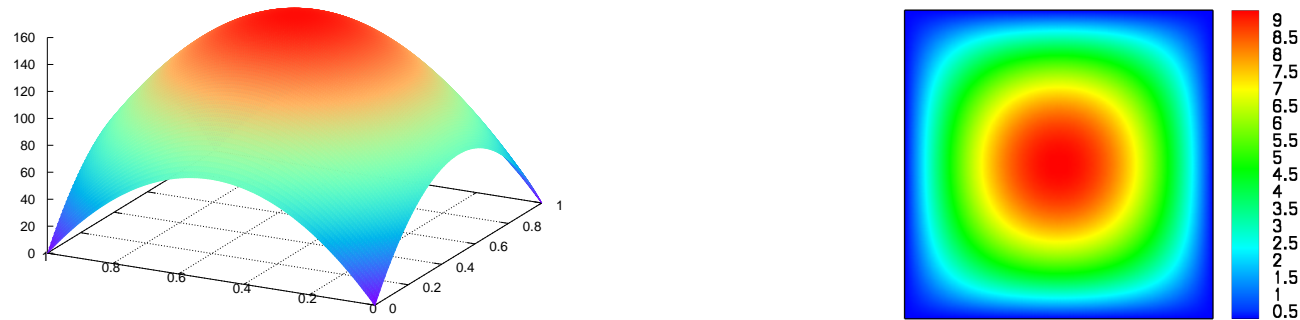

Figure 1: Source term $J_{z}^{E}$ (left) and electric field $E^{z}$ (right) at $T=5$.

We investigate here the space-time convergence order of the fully explicit method (28) and the locally implicit methods (34) and (40), for different local refinement rates, denoted by $r$. We consider a sequence of four successively refined triangular meshes (see Figure 2 for an example of a mesh with the identification of the implicit region). The characteristics of the different meshes used in the numerical tests are summarized in Table 2 for refinement rates $r=1,2,3,4$ (see also Figure 3). Note that for the fully explicit method (28) the size of the critical time step (see (64)) is determined by the smallest height of the mesh, $h^{\min }$, while for the locally implicit methods (34) and (40), it is determined by the largest height, $h^{\max }$, see Table 2 .

Now we can estimate the convergence order in the PDE sense i.e. for a stable simultaneous spacetime grid refinement $\Delta t \backsim h, h \rightarrow 0$. We measure the final $L^{2}$-norm of the error for different meshes of increased resolution and we plot this error as a function of the square root of the number of degrees of freedom (DOF), in logarithmic scale. The use of the logarithmic scale allows to visualize the convergence rates as the slopes of the curves. Note that we use the DGTD- $\mathbb{P}_{2}$ method so that the spatial error is not detrimental to the temporal convergence orders. The obtained results for the fully explicit method 28) and the locally implicit methods (34) and (40) are presented on Figure 4 For each method we observe 


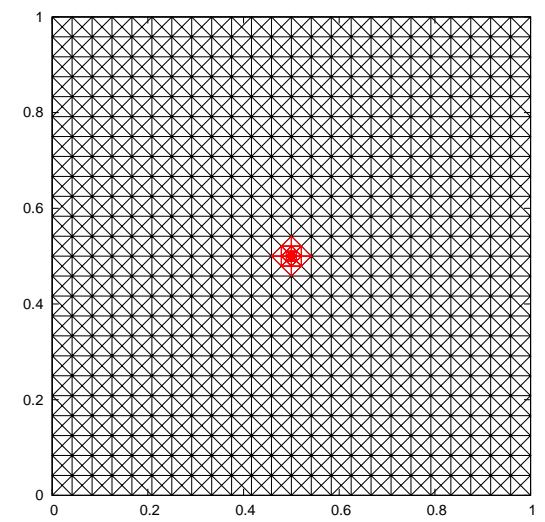

Figure 2: Example of a mesh used in numerical tests (\# elements: 2368, implicit treatment : red region).
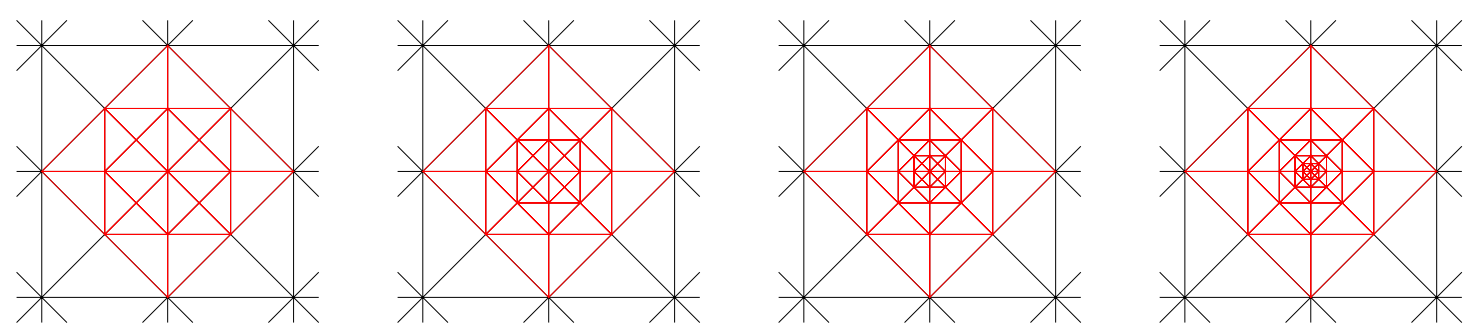

Figure 3: Rates of local refinement, from left to right $r=1,2,3$ and 4.

\begin{tabular}{|c|c|c|}
\hline \# elements & $\begin{array}{c}r=1 \\
h^{\text {min }}\end{array}$ & $h^{\max }$ \\
\hline 800 & 0.02525 & 0.07143 \\
\hline 2320 & 0.01473 & 0.04167 \\
\hline 4640 & 0.01040 & 0.02941 \\
\hline \multirow[t]{2}{*}{7760} & 0.00803 & 0.02273 \\
\hline & \multicolumn{2}{|l|}{$r=3$} \\
\hline \# elements & $h^{\min }$ & $h^{\max }$ \\
\hline 832 & 0.00631 & 0.07143 \\
\hline 2352 & 0.00368 & 0.04167 \\
\hline 4672 & 0.00260 & 0.02941 \\
\hline 7792 & 0.00201 & 0.02273 \\
\hline
\end{tabular}

\begin{tabular}{|c|c|c|}
\hline \# elements & $\begin{array}{c}r=2 \\
h^{\text {min }}\end{array}$ & $h^{\max }$ \\
\hline 816 & 0.01263 & 0.07143 \\
\hline 2336 & 0.00736 & 0.04167 \\
\hline 4656 & 0.00520 & 0.02941 \\
\hline \multirow[t]{2}{*}{7776} & 0.00402 & 0.02273 \\
\hline & \multicolumn{2}{|l|}{$r=4$} \\
\hline \# elements & $h^{\min }$ & $h^{\max }$ \\
\hline 848 & 0.00316 & 0.07143 \\
\hline 2368 & 0.00184 & 0.04167 \\
\hline 4688 & 0.00130 & 0.02941 \\
\hline 7808 & 0.00100 & 0.02273 \\
\hline
\end{tabular}

Table 2: Data of the four successively triangular meshes, for local refinements, $r=1,2,3,4$. 
a super-convergence phenomenon, i.e. a third-order convergence rate for the methods (28) and (40) instead of the expected second-order rate; similarly, we observe a second-order convergence rate for the method (34) instead of the expected first-order rate. However these results clearly confirm the reduction order by one for the locally implicit method (34) (see Section 4.1.3, Theorem 1) while the subdivision into coarse and fine elements is not detrimental for the convergence order for the locally implicit method (40) (see Section 4.2.3, Theorem 3) since we observe the same behavior for the fully explicit method (28).


Figure 4: Convergence of the fully explicit method (28) (top left) and the locally implicit methods (34) 40] (top right - bottom left, respectively).

\subsubsection{Computational work for the implicit treatment}

Now we focus on the cost of solving the linear systems of each locally implicit method. In particular, we are interested in the sparsity of the matrices to be inverted and the cost of the factorization step. Indeed, the matrices are factored once for all before the main time stepping loop. Then, each linear system inversion amounts to a forward and a backward solve using the $\mathrm{L}$ and $\mathrm{U}$ factors. All the numerical tests presented in this section have been conducted on the regular triangular mesh of the Figure 2 (\# elements : 2368, local refinement $r=4$, implicit treatment : red region). In Table 3 for each matrix of linear system to solve, we indicate the number of nonzero elements, the memory requirements for storing the $\mathrm{L}$ and $\mathrm{U}$ factors, 
and the factorization time. In order to appreciate the properties of the locally implicit methods we also reported the results of the fully implicit method (30). To further illustrate the gain in sparsity, Figure 5 shows the sparsity pattern for the matrices $\mathscr{M}, \mathscr{M}_{1}$ and $\mathscr{M}_{2}$ defined in (48), (38) and (47), respectively, based on a DGTD- $\mathbb{P}_{2}$ method. As anticipated in Section 4.2.1 we remark that if we omit the identity matrix in the matrices $\mathscr{M}_{1}$ and $\mathscr{M}_{2}$ we recover a very close number of nonzero elements.

\begin{tabular}{lllll}
\hline \multicolumn{5}{c}{ Fully implicit method } \\
$\mathbb{P}_{k}$ & Matrix order & \# nonzero $(\mathrm{nz})$ & RAM size $(\mathrm{MB})$ & CPU time (s) \\
\hline $\mathbb{P}_{1}$ & 7104 & 160125 & 33 & 0.62 \\
$\mathbb{P}_{2}$ & 14208 & 563782 & 118 & 2.05 \\
$\mathbb{P}_{3}$ & 23680 & 1457292 & 304 & 6.21 \\
$\mathbb{P}_{4}$ & 35520 & 3099475 & 634 & 12.90 \\
\hline
\end{tabular}

\begin{tabular}{lllll}
\hline \multicolumn{5}{c}{ Locally implicit method $[34): \mathscr{M}_{1}=I+\frac{\Delta t^{2}}{4} S_{i} S_{i}^{T}$} \\
$\mathbb{P}_{k}$ & Matrix order & \# nonzero (nz) & RAM size (MB) & CPU time (s) \\
\hline $\mathbb{P}_{1}$ & 216 & 4680 & 2 & 0.03 \\
$\mathbb{P}_{2}$ & 432 & 16380 & 4 & 0.07 \\
$\mathbb{P}_{3}$ & 720 & 42329 & 7 & 0.17 \\
$\mathbb{P}_{4}$ & 1080 & 90121 & 14 & 0.36 \\
\hline
\end{tabular}

\begin{tabular}{lllll}
\hline \multicolumn{5}{c}{ Locally implicit method } \\
$\mathbb{P}_{k}$ & Matrix order & \# nonzero $(\mathrm{nz})$ & $\mathscr{M}_{2}=I+\frac{\Delta t^{2}}{4} S_{1} S_{1}^{T}$ & \\
\hline $\mathbb{P}_{1}$ & 7104 & 11832 & 5 & RAM size (MB) \\
$\mathbb{P}_{2}$ & 14208 & 30996 & 10 & 0.33 \\
$\mathbb{P}_{3}$ & 23680 & 67347 & 17 & 0.95 \\
$\mathbb{P}_{4}$ & 35520 & 128740 & 27 & 2.86 \\
\hline
\end{tabular}

Table 3: Data and factorization of the matrix of the linear system to be solved for the fully implicit method (30) and the locally implicit methods (34) - (40), with DGTD- $\mathbb{P}_{k}$ methods $(k=1,2,3,4)$.

\subsubsection{Comparison of locally implicit methods and the fully explicit method}

Finally we present some numerical results for the locally implicit methods and the fully explicit method (28) on the regular mesh of Figure 2 with a local refinement $r=4$. In Table 4 we observe that the locally implicit methods allow to overcome the step size limitations caused by the local refinement. The obtained results are very close for the two locally implicit methods (34) and (40). With implicit-explicit approaches the sizes of the time step are about 16 times larger which yields significant gains of final CPU time, about 8 times lower than the fully explicit case. Regarding the error (see Figure 6) we observe that the locally implicit scheme (40) and the fully explicit scheme (28) based on DGTD- $\mathbb{P}_{1,2,3}$ methods give similar results. For a DGTD- $\mathbb{P}_{4}$ method the difference is more pronounced; we can assume that in the latter case the error in time is predominant on the error in space. As expected Figure 6 also confirms that the locally implicit method (34) is less accurate. Because of the reduction by one of the order of convergence, a 

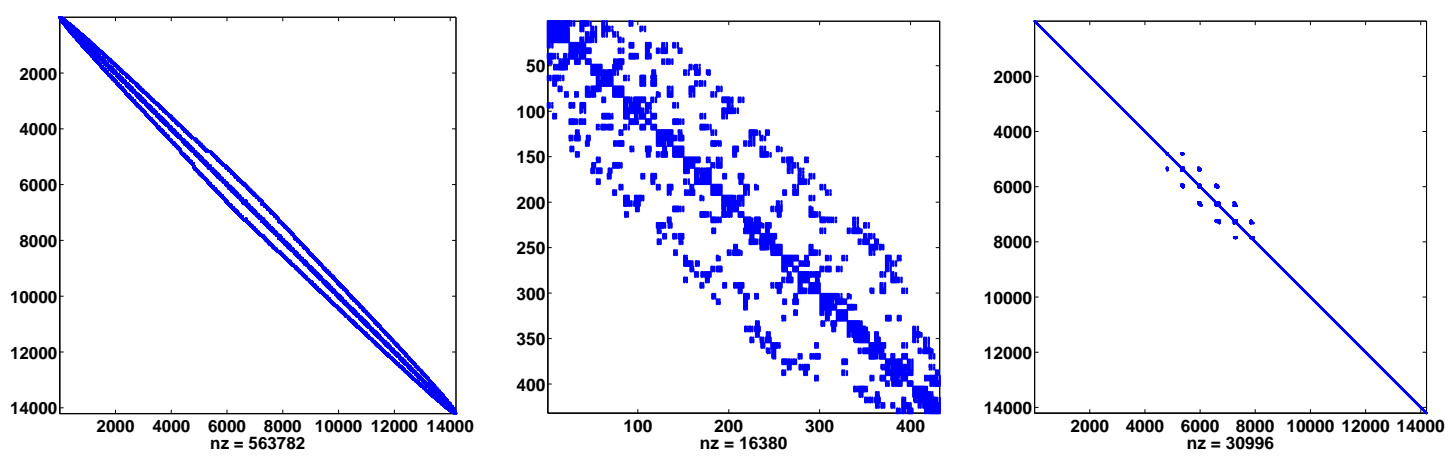

Figure 5: Sparsity pattern of the matrix of the linear system to solve for the fully implicit method (30) and the locally implicit methods (34) - (40) (from left to right), with a DGTD- $\mathbb{P}_{2}$ method.

high order spatial discretization is less advantageous for method (34) than for method (40) which retain its second-order PDE convergence. However by comparing the different levels of error and taking into account the gain in time compared to the fully explicit method, the errors of the method 34 remain very acceptable.

\begin{tabular}{|c|c|c|c|c|c|c|c|c|}
\hline \multirow[b]{2}{*}{$\mathbb{P}_{k}$} & \multicolumn{2}{|c|}{ Fully exp. method 28) } & \multicolumn{3}{|c|}{ Loc. imp. method 34 } & \multicolumn{3}{|c|}{ Loc. imp. method 40 } \\
\hline & $\Delta t$ & $\mathrm{CPU}$ & $\Delta t$ & $\mathrm{CPU}$ & $\frac{\mathrm{CPU}_{28}}{\mathrm{CPU}_{34}}$ & $\Delta t$ & $\mathrm{CPU}$ & $\frac{\mathrm{CPU}_{28}}{\mathrm{CPU}_{40}}$ \\
\hline $\mathbb{P}_{1}$ & $3.91 \mathrm{e}-4$ & 48.4 & $6.25 \mathrm{e}-3$ & 10.4 & 4.7 & $6.25 \mathrm{e}-3$ & 6.8 & 7.2 \\
\hline $\mathbb{P}_{2}$ & $2.60 \mathrm{e}-4$ & 202.3 & $4.17 \mathrm{e}-3$ & 27.5 & 7.4 & $4.17 \mathrm{e}-3$ & 25.2 & 8.1 \\
\hline $\mathbb{P}_{3}$ & $1.69 \mathrm{e}-4$ & 731.7 & $2.71 \mathrm{e}-3$ & 84.2 & 8.7 & $2.71 \mathrm{e}-3$ & 87.4 & 8.4 \\
\hline $\mathbb{P}_{4}$ & $1.17 \mathrm{e}-4$ & 2328.4 & $1.87 \mathrm{e}-3$ & 241.5 & 9.7 & $1.87 \mathrm{e}-3$ & 257.4 & 9.1 \\
\hline
\end{tabular}

Table 4: Critical time step size and CPU time for the fully explicit method (28) and the locally implicit methods (34) - 40, with DGTD- $\mathbb{P}_{k}$ methods $(k=1,2,3,4)$.

The numerical results presented in this section clearly show that, for a workload per time step about equal, the IMEX method (40) is more accurate than the IMEX method (34). Consequently, for the next numerical test case, we will focus on the method 40.).

\subsection{Scattering of an airfoil profile}

We consider an electromagnetic wave propagating into a rectangular domain $\Omega=[-1,2] \times[-1,1]$, with an airfoil profile, see Figure 7 We set $\varepsilon=\mu=1$ and $\sigma=0$ in 63, and we impose an absorbing boundary condition on the boundary of the domain (with a zero incident field) and a metallic boundary condition on the boundary of the airfoil profile, see (2). The electromagnetic field is excited by a local source term

$$
J_{z}^{E}(x, y, t)=f(t) g(x, y),
$$

where $f$ is a time dependent function (Figure 8), $g$ is a two-dimensional Gaussian function with $\left(x_{0}, y_{0}\right)=$ $(-0.3,0.0)$ the center of the Gaussian spatial support

$$
g(x, y)=A e^{-\alpha\left(\left(x-x_{0}\right)^{2}+\left(y-y_{0}\right)^{2}\right)} .
$$



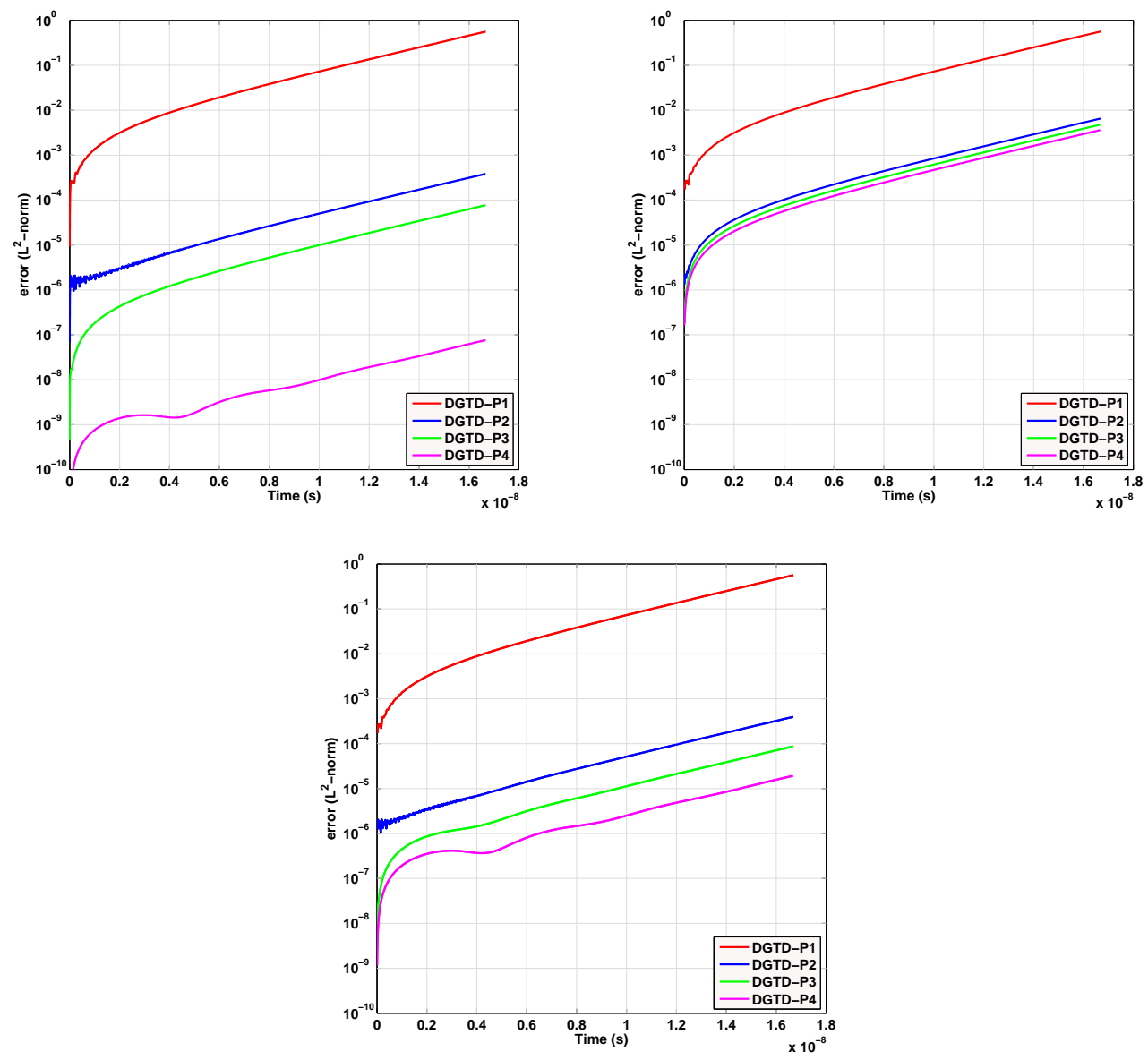

Figure 6: Time evolution of the error (in $L^{2}$-norm) for the fully explicit method 28] (top left) and the locally implicit methods (34) - 40) (top right - bottom left, respectively), with DGTD- $\mathbb{P}_{k}$ methods $(k=$ $1,2,3,4)$. 
For the numerical experiments, $A=1 /\|g\|$ and the parameter $\alpha$ has been chosen such that the source term $J_{z}^{E}$ is strongly localized, which justifies the local refinement around the center of the Gaussian (the support of the Gaussian $g$ is strictly included in the implicit zone, see Figures 7 and 8). Figure 9 shows the intensity of the electric field at different times.
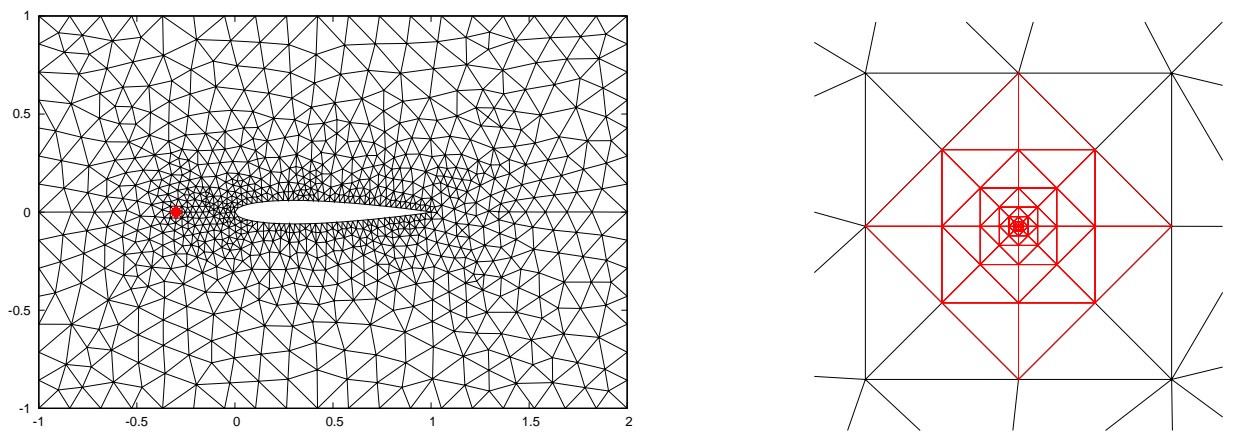

Figure 7: The mesh used in numerical tests with the airfoil profile (\# elements: 1817, implicit treatment : red region).
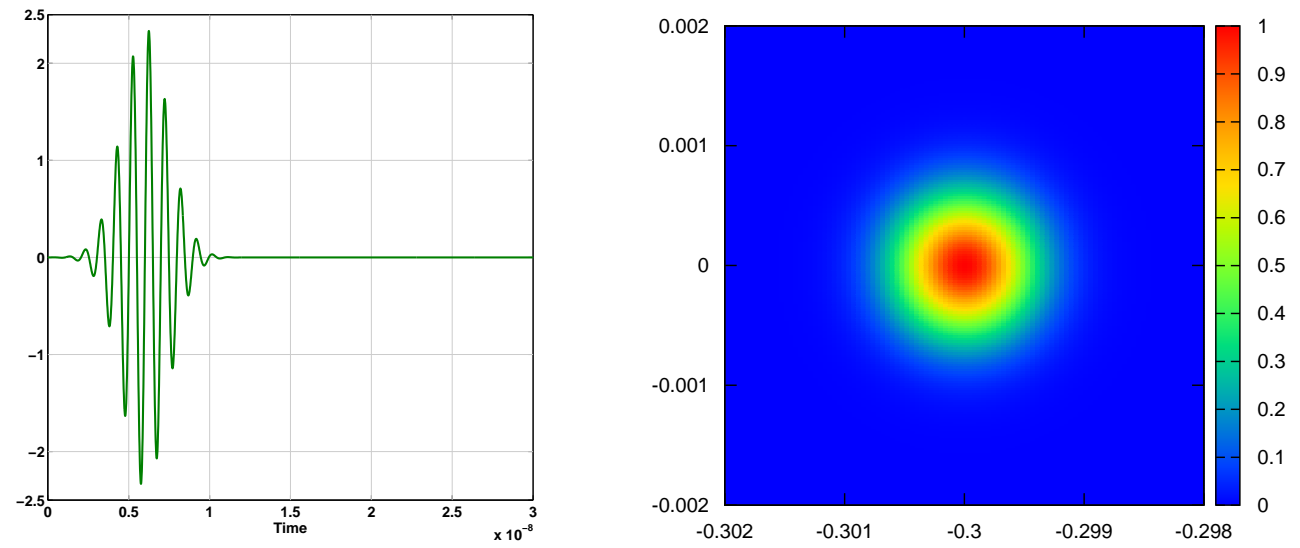

Figure 8: Temporal signal $f(t)$, and Gaussian function $g(x, y) / A$ on $[-0.302,-0.298] \times[-0.002,0.002]$.

Similarly to the previous test case we measure the cost of solving the linear systems by indicating in Table 5 the number of nonzero elements and the memory requirement for the factorization of the matrix. We observe again significant gains with the locally implicit method (40) compared to the fully implicit method. Finally, with the numerical results reported in Table 6 for a total simulation time $T=9$, we can observe that the locally implicit approach allows to overcome the severe stability constraints imposed on the fully explicit method by the local refinement; the sizes of the time step are about 26 times larger. This yields significant gains in terms of the CPU time (the IMEX method is 12.5 times faster on average). Furthermore, the Figure 10 shows similar results for the locally implicit method (40) and the explicit method (28) regardless of the degree of interpolation in the DG method. These results clearly confirm the high accuracy of the IMEX method (40). 

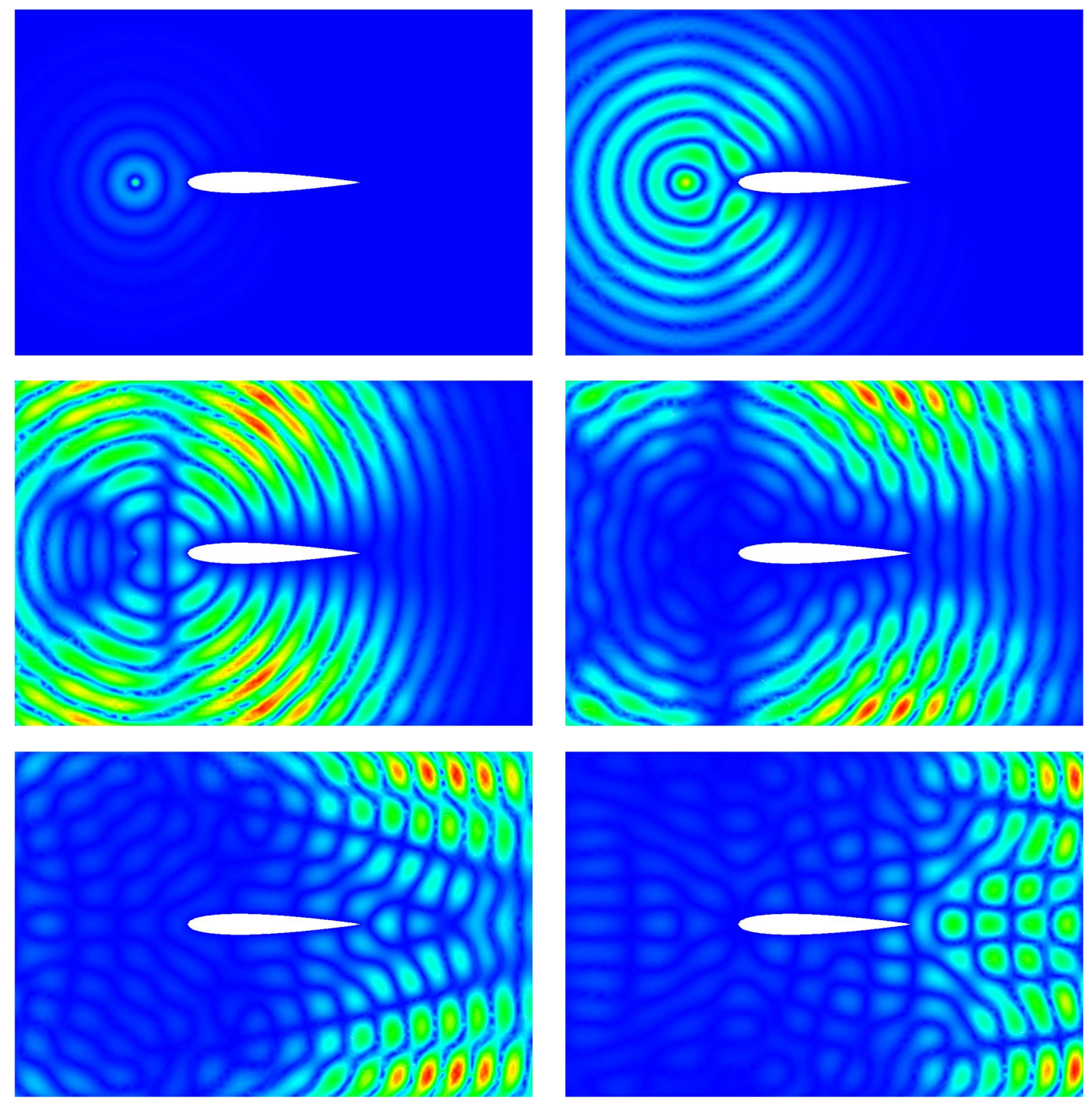

Figure 9: Intensity of the electric field, $\left|E_{z}\right|$, at time $t=1.5,2.3,3.0,3.5,4.0$ and 4.5.

\begin{tabular}{|c|c|c|c|c|c|c|}
\hline \multirow[b]{2}{*}{$\mathbb{P}_{k}$} & \multirow[b]{2}{*}{ Matrix order } & \multicolumn{2}{|c|}{ Fully imp. method $30 p$} & \multicolumn{2}{|c|}{ Loc. imp. method $(40)$} & \multirow{2}{*}{$\begin{array}{l}\text { Gain } \\
n z_{30} / \mathrm{nz}_{40}\end{array}$} \\
\hline & & $\# \mathrm{nz}$ & $\mathrm{RAM}(\overrightarrow{\mathrm{MB}})$ & $\# \mathrm{nz}$ & RAM (MB) & \\
\hline $\mathbb{P}_{1}$ & 5451 & 125349 & 28 & 11043 & 5 & 11.3 \\
\hline $\mathbb{P}_{2}$ & 10902 & 435966 & 99 & 30640 & 9 & 14.2 \\
\hline $\mathbb{P}_{3}$ & 18170 & 1117633 & 243 & 69967 & 16 & 16.0 \\
\hline $\mathbb{P}_{4}$ & 27255 & 2365453 & 498 & 138185 & 28 & 17.1 \\
\hline
\end{tabular}

Table 5: Data and factorization of the matrix of the linear system to solve for the fully implicit method (30) and the locally implicit method (40), with DGTD- $\mathbb{P}_{k}$ methods $(k=1,2,3,4)$. 


\begin{tabular}{llllll}
\hline & \multicolumn{2}{l}{ Fully exp. method $[28)$} & \multicolumn{2}{l}{ Loc. imp. method $[40)$} & \multicolumn{2}{l}{ Gain } \\
$\mathbb{P}_{k}$ & $\Delta t$ & $\mathrm{CPU}$ & $\Delta t$ & $\mathrm{CPU}$ & $\mathrm{CPU}_{[28} / \mathrm{CPU}_{[40]}$ \\
\hline $\mathbb{P}_{1}$ & $1.40 \mathrm{e}-4$ & 202.1 & $3.62 \mathrm{e}-3$ & 18.6 & 10.9 \\
$\mathbb{P}_{2}$ & $9.36 \mathrm{e}-5$ & 878.9 & $2.41 \mathrm{e}-3$ & 73.9 & 11.9 \\
$\mathbb{P}_{3}$ & $6.08 \mathrm{e}-5$ & 3297.9 & $1.57 \mathrm{e}-3$ & 250.3 & 13.2 \\
$\mathbb{P}_{4}$ & $4.21 \mathrm{e}-5$ & 10810.7 & $1.11 \mathrm{e}-3$ & 764.0 & 14.1 \\
\hline
\end{tabular}

Table 6: Critical time step size and CPU time for the fully explicit method (28) and the locally implicit method 40, with DGTD- $\mathbb{P}_{k}$ methods $(k=1,2,3,4)$.
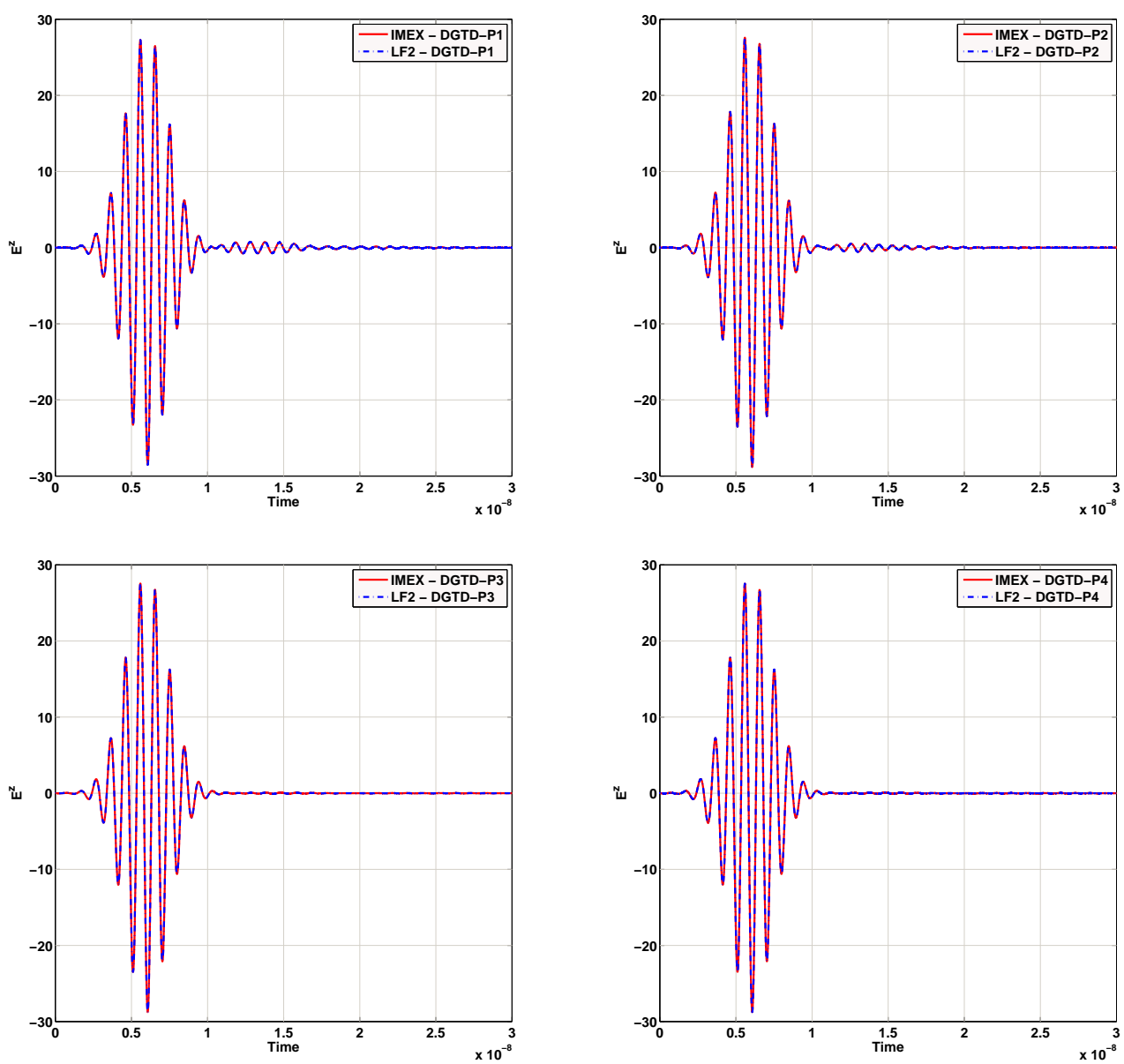

Figure 10: Time evolution of the electric field, $E^{z}$, at point $(x, y)=(-0.298,0.0)$, for the fully explicit method $(28)$ and the locally implicit method $[40)$, with DGTD- $\mathbb{P}_{k}$ methods $(k=1,2,3,4)$. 


\section{Conclusion}

In this paper we have considered two locally implicit time integration approaches from [17] and [21], respectively, for the time-dependent Maxwell equations spatially discretized with a DG method. In the presence of a local refinement, we have seen that the purpose of the two IMEX methods is achieved, i.e. the most severe stability constraints on explicit time integration methods are overcome. By adjusting the splitting, we have highlighted the similarities between the two schemes (34) and (40). However, while the second method is more accurate because, unlike the first method, it does not suffer from order reduction, it is also more implicit, regarding the dimension and the sparsity of the matrice of the linear system to be solved. By comparing the two approaches for a two-dimensional problem (see the first test case), we find that the overhead for solving the linear system by a direct method using the LU decomposition is not excessive. Consequently, for two-dimensional problems, we can advise the use of the IMEX method from [21]. Regarding numerical experiments in three dimensions, this issue deserves further analysis; iterative methods may be more suitable for solving the linear system which could lead to extra solution costs for the method (40) compared to the method (34). In the near future we plan to carry out such comparison.

Another plan is to establish a numerical comparison between the two IMEX approaches and an explicit local time-stepping method, which also allows to overcome step size limitation induced by local refinements. For example, the methods from [10, 17, 19] which are also designed for the time-domain Maxwell equations spatially discretized with a DG method, are possible candidates.

\section{References}

[1] U.M. Ascher, S.J. Ruuth and R.J. Spiteri, Implicit-explicit Runge-Kutta methods for time-dependent partial differential equations, Appl. Numer. Math. 25 (1997) 151-167.

[2] M.A. Botchev and J.G. Verwer, Numerical Integration of Damped Maxwell Equations, SIAM J. SCI. Comput. 31 (2009) 1322-1346.

[3] M.P. Calvo, J. de Frutos and J. Novo, Linearly implicit Runge-Kutta methods for advection-reactiondiffusion equations, Appl. Numer. Math. 37 (2001) 535-549.

[4] B. Cockburn, G.E. Karniadakis and C.-W. Shu (Eds.), Discontinuous Galerkin methods. Theory, computation and applications. Springer-Verlag, Berlin (2000).

[5] M. Crouzeix, Une méthode multipas implicite-explicite pour l'approximation des équations d'évolution paraboliques, Numer. Math. 35 (1980) 257-276.

[6] J. Diaz and M. Grote, Energy conserving explicit local time-stepping for second-order wave equations, SIAM J. SCI. Comput. 31 (2009) 1985-2014.

[7] V. Dolean, H. Fahs, L. Fezoui and S. Lanteri, Locally implicit discontinuous Galerkin method for time domain electromagnetics, J. Comput. Phys. 229 (2010) 512-526.

[8] H. Fahs, Development of a hp-like discontinuous Galerkin time-domain method on non-conforming simplicial meshes for electromagnetic wave propagation, Int. J. Numer. Anal. Mod. 6 (2009) 193-216.

[9] L. Fezoui, S. Lanteri, S. Lohrengel and S. Piperno, Convergence and stability of a discontinuous Galerkin time-domain method for the 3D heterogeneous Maxwell equations on unstructured meshes, ESAIM: M2AN 39 (2005) 1149-1176. 
[10] M.J. Grote and T. Mitkova, Explicit local time stepping methods for Maxwell's equations, J. Comp. Appl. Math. 234 (2010) 3283-3302.

[11] J. Hesthaven and T. Warburton, Nodal high-order methods on unstructured grids. I. Time-domain solution of Maxwell's equations, J. Comput. Phys. 181 (2002) 186-221.

[12] J.S. Hesthaven and T. Warburton, Nodal Discontinuous Galerkin Methods, Springer (2008).

[13] A. Kanevsky, M.H. Carpenter, D. Gottlieb and J.S. Hesthaven, Application of implicit-explicit high order Runge-Kutta methods to discontinuous-Galerkin schemes, J. Comput. Phys. 225 (2007) 17531781.

[14] C.A. Kennedy and M.H. Carpenter, Additive Runge-Kutta schemes for convection-diffusionreaction equations, Appl. Numer. Math. 44 (2003) 139-181.

[15] E. Montseny, S. Pernet, X. Ferrières and G. Cohen, Dissipative terms and local time-stepping improvements in a spatial high order Discontinuous Galerkin scheme for the time-domain Maxwell's equations, J. Comput. Phys. 227 (2008) 6795-6820.

[16] L. Moya, Temporal convergence of a locally implicit discontinuous Galerkin method for Maxwell's equations, ESAIM: M2AN, 46 (2011) 1225-1246.

[17] S. Piperno, Symplectic local time-stepping in non-dissipative DGTD methods applied to wave propagation problem. ESAIM: M2AN 40 (2006) 815-841.

[18] W.H. Reed and T.R. Hill, Triangular mesh methods for the neutron transport equation, Los Alamos Scientific Laboratory Report LAUR-73-479, 1973

[19] A. Taube, M. Dumbser, C.D. Munz and R. Schneider, A high order discontinuous Galerkin method with local time stepping for the Maxwell equations, Int. J. Numer. Model.: Elec. Netw. Dev. and Fields 22 (2009) 77-103.

[20] J.M. Varah, Stability restrictions on second, three- level finite-difference schemes for parabolic equations, SIAM J. Numer. Anal. 17 (1980) 300-309.

[21] J.G. Verwer, Component splitting for semi-discrete Maxwell equations, BIT Numer. Math. (2010) DOI 10.1007/s10543-010-0296-y.

[22] J.G. Verwer and M.A. Botchev, Unconditionaly stable integration of Maxwell's equations. Linear Algebra and its Applications 431 (2009) 300-317.

[23] P. Wesseling, Principles of Computational Fluid Dynamics. Springer Series in Computational Mathematics, Vol. 29, Springer, Berlin (2001).

[24] K.S Yee, Numerical solution of initial boundary value problems involving Maxwell's equations in isotropic media, IEEE Trans. Antenn. Propag. 14 (1966) 302-307. 


\section{Contents}

\begin{tabular}{llr}
\hline & Introduction & 3 \\
\hline
\end{tabular}

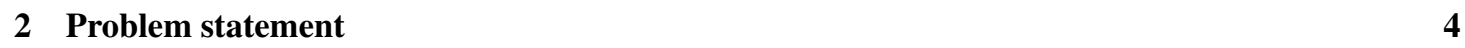

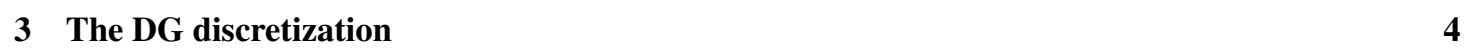

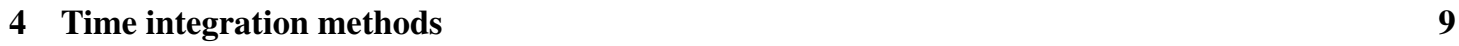

4.1 The locally implicit method from [17] . . . . . . . . . . . . . . . . . . . . . . . . 10

4.1 .1 Computational work . . . . . . . . . . . . . . . . . . . . . . . . . . . . . . . . .

4.1.2 Stability and conservation properties . . . . . . . . . . . . . . 11

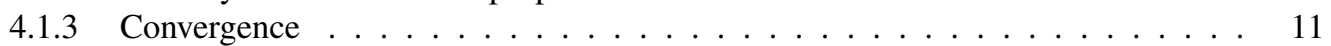

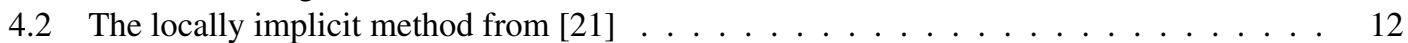

4.2 .1 Computational work . . . . . . . . . . . . . . . . . . 12

4.2 .2 Stability and conservation properties . . . . . . . . . . . . . . . . . . . . . . . . . . . . . . . 14

4.2 .3 Convergence $\ldots \ldots \ldots \ldots \ldots \ldots$

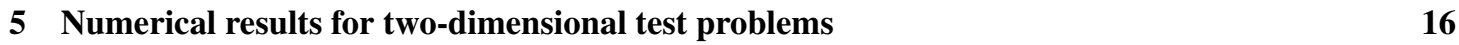

5.1 2D TM Maxwell's equations $\ldots \ldots \ldots \ldots \ldots \ldots$

5.2 Model test problem with an exact solution . . . . . . . . . . . . . . . . . . . . . 17

5.2.1 Computational work for the implicit treatment . . . . . . . . . . . . . . 19

5.2.2 Comparison of locally implicit methods and the fully explicit method . . . . . . 20

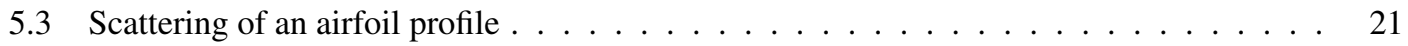

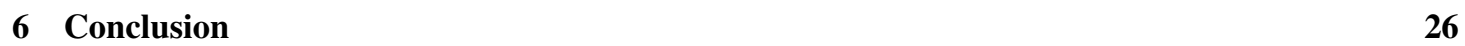




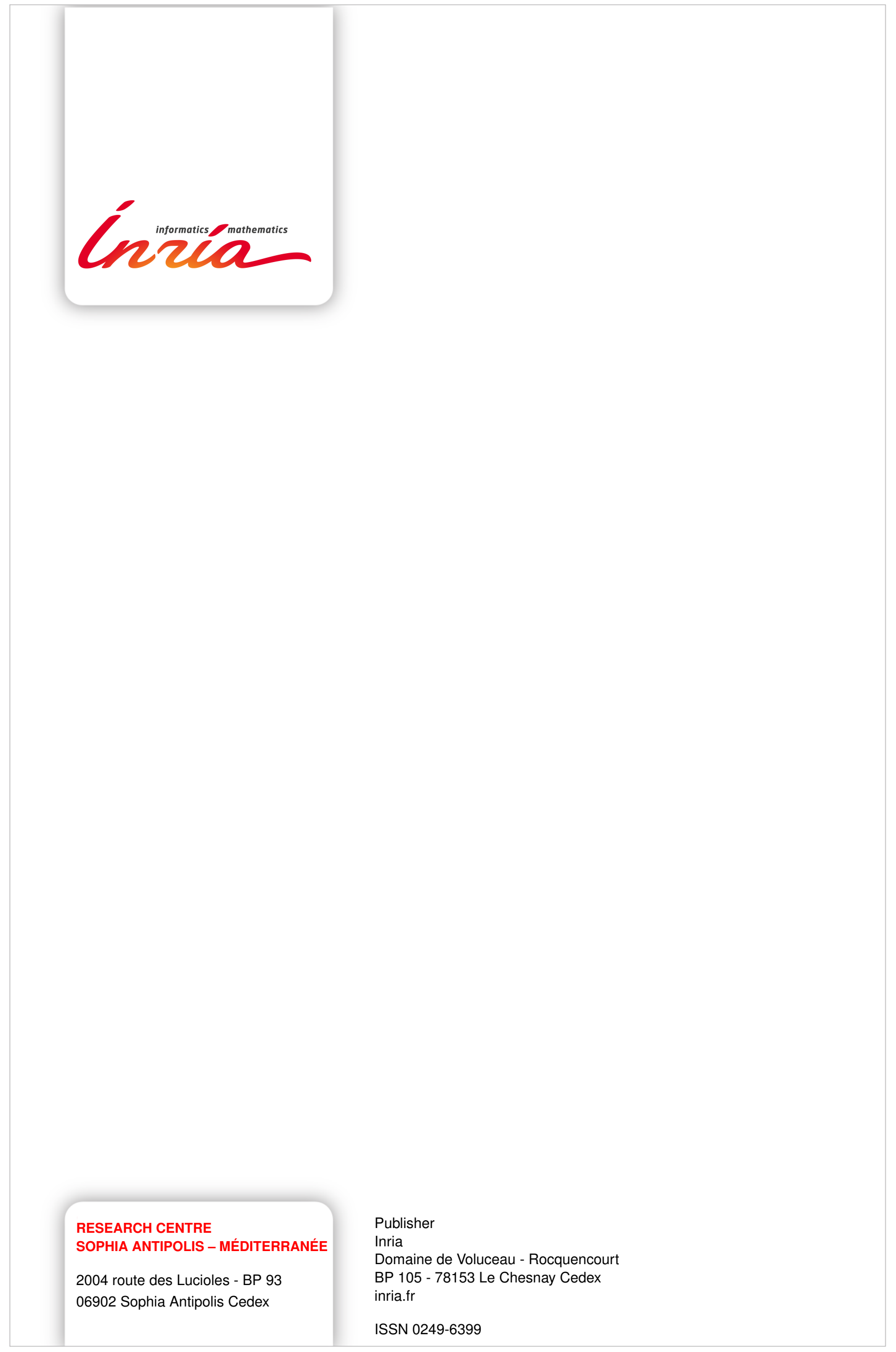

